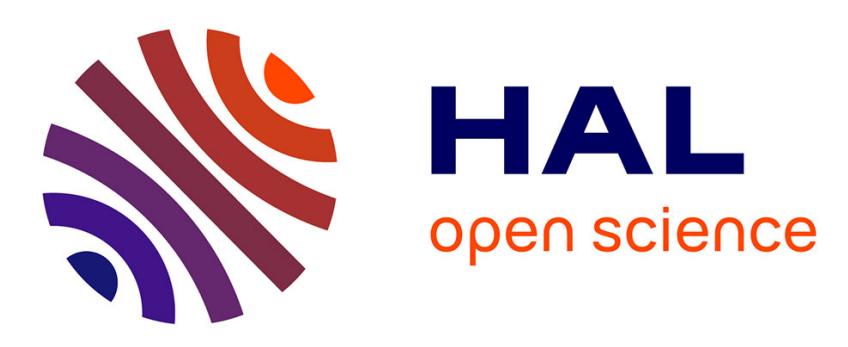

\title{
Self-regulation of turbulence bursts and transport barriers
}

Elena Floriani, Guido Ciraolo, Philippe Ghendrih, Yanick Sarazin, Ricardo Lima

\section{- To cite this version:}

Elena Floriani, Guido Ciraolo, Philippe Ghendrih, Yanick Sarazin, Ricardo Lima. Self-regulation of turbulence bursts and transport barriers. Plasma Physics and Controlled Fusion, 2013, 55 (9), pp.095012. 10.1088/0741-3335/55/9/095012 . hal-00733279

\section{HAL Id: hal-00733279 \\ https://hal.science/hal-00733279}

Submitted on 18 Sep 2012

HAL is a multi-disciplinary open access archive for the deposit and dissemination of scientific research documents, whether they are published or not. The documents may come from teaching and research institutions in France or abroad, or from public or private research centers.
L'archive ouverte pluridisciplinaire HAL, est destinée au dépôt et à la diffusion de documents scientifiques de niveau recherche, publiés ou non, émanant des établissements d'enseignement et de recherche français ou étrangers, des laboratoires publics ou privés. 


\title{
Turbulence bursts probing of transport barriers analyzed in terms of competing stochastic processes
}

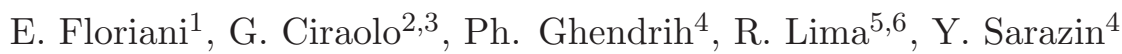

1) Aix-Marseille Univ, Centre de Physique Théorique, 13009 Marseille, France.

2) Centrale Marseille, 13451 Marseille, France.

3) Aix Marseille Univ, Mécanique, Modélisation et Procédés Propres (M2P2) - 13451 Marseille, France.

4) CEA, IRFM, F-13108 Saint-Paul-lez-Durance, France.

5) Dream \& Science Factory, Marseille, France.

6) Institute for Complexity Sciences (ICC), Lisbon, Portugal.

\begin{abstract}
The interplay between turbulent bursts and transport barriers in analyzed with a simplified model of interchange turbulence in the Scrape-Off Layer of magnetically confined plasmas. The turbulent bursts spread into the transport barriers, and, depending on the competing magnitude of the burst and stopping capability of the barrier can burn through. Two models of transport barriers are presented, a hard barrier where all turbulent modes are stable in a prescribed region and a soft barrier with external plasma biasing. This process can be modeled on the basis of competing stochastic processes. For classes of probability density function of these processes one can predict the heavy tail properties of the bursts downstream from the barrier, either exponential for a leaky barrier, or with power laws, for a tight barrier. The intrinsic probing of the transport barriers by the turbulent bursts thus gives access to properties of the transport barriers. The main stochastic variables of the two models addressed here are the barrier width and the spreading distance of the turbulent bursts within the barrier together with their level of correlation. One finds that in the case of a barrier located in the Scrape-Off-Layer, the stochastic model predicts a leaky barrier with an exponential probability density function of escaping turbulent bursts in agreement with the simulation data.
\end{abstract}

\section{Introduction}

Transport barriers are key confinement structures in all phases of ITER operation [1], both during the initial phase with an Edge Transport Barrier (ETB or H-mode) [2] to achieve a significant energy amplification factor [1], and during the second stage with the possible build-up of Internal Transport Barriers (ITB) to achieve steady-state operation [3]. Although experimental procedures to establish such transport barriers are followed routinely in present day tokamaks, there is to date no complete theoretical description for the generation of such self-organized structures. Consequently, the predictive capability for the onset and behavior of the transport barrier is only based on empirical data. In such a process, one thus is led to assume that large classes of these barriers are governed by the same law and consequently are considered to be identical despite significant experimental scatter. As a unifying paradigm, it is however agreed that the shearing of turbulence eddies by large scale flows, transverse to the main direction of propagation source $\leftrightarrow \operatorname{sink}$, is a key process [4]. With the development 
of new diagnostics, it is now possible to improve the description and understanding of these barriers $[5,6]$. In this paper we propose to use the fact that the turbulent bursts or avalanches probe the barriers spontaneously. The statistical analysis we propose for such a probing then allows one to interpret the Probability Density Functions (PDFs) obtained either in simulations or experimentally. In particular, this provides a means to quantify the quality of transport barriers.

The complexity of plasma turbulence and the difficulty to consistently measure relevant parameters that govern transport properties has led to a rather stiff representation of transport barriers [7, 8]. External transport barriers are most often viewed as steady-state structures that exhibit quasi-periodic collapses due to either of the various types of $E d g e$ Localized Modes. Conversely, internal transport barriers appear to be characterized by adiabatic changes in particular regarding their location. Furthermore, the attention driven by the performance of the most developed transport barriers that are required for ITER has dwarfed the interest for the zoology of transport barriers that are experimentally achieved. A first set of the sort appears to be related to moderate machine conditioning. Such transport barriers mainly act on the density field, with ELMs [9] or ELM free [10]. Conversely, high machine conditioning together with low collisionality operation has led to the VH-mode as reported on DIII-D [11]. There, the confinement improvement appears to extend significantly beyond the narrow radial region of the transport barrier [12]. Finally, within the bifurcation hysteresis of the standard ETB, there seems to be a gradual improvement of confinement [13] as the power crossing the separatrix is scanned from the threshold value to twice the threshold value. The variety of experimental observations is even more important for ITBs and no reference scenario reproducible on most devices appears to have been defined. The existence of different driving mechanisms, as widely accepted as a difference between ETBs and ITBs, can explain such a situation. Alternatively, a single mechanism driven at different amplitudes could still be considered for the ETBs.

From the theoretical point of view various aspects of turbulence control have been developed. When considering eddy shearing as the key mechanism to control turbulent transport [14], one can describe the effect as a change in the saturation level of turbulence as well as a possible shift of the turbulence linear threshold. Furthermore these effects can be viewed as purely local mechanisms or as more global effects hence including turbulence spreading as a key paradigm.

A comprehensive description of the interplay between turbulence and the various transport barriers is beyond the scope of the paper. We want here to concentrate on two important features of that problem that are characterized by interesting properties for the experimental analysis. As readily observed in global simulations, turbulent transport appears to be strongly related to self organized turbulent structures, also reported as avalanches, that propagate with ballistic motions predominantly in the radial direction. The related radial scales are intermediate between the turbulence correlation length and the macroscopic scale, namely the device minor radius [15]. Experimental evidence of such events in the plasma core have also been reported and an extensive "blob" literature has been published for SOL observations $[16,17,18]$. It is very important to underline that avalanche like transport are reported in gyrokinetic simulations of the core plasma $[19,20]$ and that there is experimental evidence of such events in the region of closed field lines with temperature measurements [23] as well as visible light imaging [24]. The idea followed here is to use such events to probe the transport barriers and thus determine interesting signatures regarding their stopping capability. Based on statistical analysis of the data one can then determine strong versus leaky barriers. Another feature of transport barriers of interest is the time dependence of these structures. While the ELMs provide an unquestionable evidence of meso-time scale variations, nor the macro-scale evolution of the transport barriers nor the micro-scale fluctuations have been given much attention. In the present paper we concentrate on the possible occurrence of micro-scale fluctuations that can also provide meaningful and direct information on the efficiency of the transport barrier. 
The paper is organized as follows. We first present simulation evidence of the dynamics of transport barriers and their interplay with the turbulence bursts, Section 2. We then introduce two models based on stochastic processes, Section 3. They allow one to compute key features of the statistics of the transport events. Indeed, statistics of these events are easier to determine experimentally and in simulations but the interpretation of their Probability Density Functions (PDF) is far more demanding. The models based on these stochastic processes provide both a guide line to select appropriate quantities to investigate and to interpret the PDFs. The mathematical aspects of these models are developed in Appendices A and B for completeness of the presentation. Finally, in Section 4, we analyze the simulation evidence on the basis of these models. Discussion and conclusion close the paper, Section 5 .

\section{Transport barriers response to turbulent burst pene- tration in numerical simulations}

\subsection{Self-organized transport events and their interplay with trans- port barriers}

In the ongoing effort to understand and control turbulent transport, it is particularly important to investigate the interplay between turbulent events that propagate from the sources to the sinks, and flows that propagate in the transverse direction with respect to the sourcesink direction. While the former drive the transport, the latter may inhibit the turbulent transport thus leading to competing effects with complex behavior. Before analyzing such effects in simulations in the next sections, it is important to recall the mechanisms that are at play. Turbulence is described by two apparently contradictory aspects, namely the occurrence of self-organized structures, turbulent eddies or vortices, together with that of a disordered media. On the one hand one addresses therefore turbulent fields in the framework of fluctuations both quasi-homogeneous and quasi-isotropic in the transverse plane. The eddy shearing stabilization has then been very successfully taken into account by computing a characteristic growth rate governed by the shear rate of the electric field [21], and which competes with the actual linear growth rate [22]. On the other hand self organized structures and avalanches are considered. When taking into account their non-local effects, alternative stabilizing effects are proposed, either a change in the saturation level, or an evolution governed by two asymptotic limits. On short time the linear growth rate can govern the linear instability, while on long times a cubic function of time can govern the asymptotic stabilization. The latter Dupree effect is directly connected to the expansion of the structure and to the effect of shearing. Such a mechanism is described in a linear framework with respect to the fluctuations and thus address the asymptotic behavior of the growth rate of the linearized system. However, the generic features of turbulence bursts in magnetic fusion plasmas appear to be non-linear structures. Avalanche-like events [16, 23, 24], and the socalled blobs [17] are "steady-state" structures. By steady-state we understand here that the structure can be identified and followed in time ans space [25, 26]. During its lifetime it can however experience significant changes in geometry and other characteristics but not to the point that it can no longer be identified. In simulations, these effects are more pronounced in global flux driven simulations. Both fluid models and kinetic models exhibit transport in bursts $[16,19]$ and these self-organized transport structures can be responsible for most of the turbulent transport [27]. They also appear to be intermittent when considering their time pattern. The PDFs of the plasma density that characterize these turbulent regimes depart significantly from Gaussians [27]. In particular the gradients of the various fields are skewed, reminiscent of lognormal distributions [28]. For the SOL, a significant effort has been made to describe the turbulent transport governed by such bursts both experimentally and theoretically [18].

When considering turbulent bursts as self-organized non-linear structures one departs 
from the very successful description of plasma turbulence in terms of waves to address transport as a sequence events. This description of plasma turbulence has already been introduced for magnetically confined plasmas for near marginal states [29]. Of particular interest for the present paper is the introduction of transport by both outgoing "overdense" structures (bump), and ingoing "subdense" structures (void), or holes. Both types of events leading to a net outflux. Recent experimental investigation in a tokamak plasma appear to support this approach [30]. Such an approach fits in the more general concept of self-organized criticality [31] where the mechanism of charging and discharging drives a stochastic response of the system. The charging aspect is readily associated to the existence of a critical gradient (hence the importance of near marginality systems) associated to a random behavior of the source. This aspect appears to be generated by the bursts of turbulent transport that also govern the discharge aspect. The understanding of such generic mechanisms in terms of stochastic processes has already been very successfully applied to solar flares where the dynamics of laminar phases are shown to generate power law distributions that are reminiscent of observations [32].

Let us now describe the structure of such turbulent bursts and their interaction with a barrier [27, 33, 34]. For consistency with the model used in this paper, we shall consider electrostatic turbulence due to interchange $[16,35]$. For simplicity we only describe the situation in the low field side midplane of a toroidal device. The interchange driven current is then in the poloidal direction, transverse to the mean transport in the radial direction. The mechanisms that drives these structures is based on a pressure front such that the gradients at the boundaries are large enough to generate a dipolar electric potential structure (governed by the interchange current). The pressure front is then localized between a local minimum and a local maximum of the electric potential $[16,27,33,34]$. In this location, the electric drift flow transports the pressure front radially which in turn drags the electric potential dipole. The velocity of the turbulent burst is proportional to the poloidal gradient of the electric potential in the dipolar structure. Such a simplified description of the motion of these structures is readily found in [17]. In the turbulent regime these turbulent structures can be tracked with pattern recognition tools $[25,26]$.

The transport barrier is a negative or positive bell shaped peak of the electric potential localized radially and extending in the poloidal direction [27, 33, 34]. The electric drift governed by the pattern of the electric potential is then poloidal and sheared. It reverses at the peak of the bell shape. As reported in previous papers, the effect of such a barrier on the turbulent burst is to extend it poloidally, as readily expected due to the poloidal flow generated by such barriers. In a first approximation for an incompressible transverse velocity field, the surface of the structure in nearly constant. The consequences of this change in geometry are two-fold, first the radial velocity of the structure is strongly reduced due to increased distance between the electric potential minimum and maximum, second the structure is squeezed, so that its radial extent is strongly reduced while its perimeter increases significantly, as sketched in figure 1. The model used to illustrate the barrier effect in 1 is given by the evolution of a structure (typically a circular structure at time zero) with two independent velocity fields, a radial velocity and a poloidal velocity [34]. The model approximately conserves the area and exemplifies the behavior of a turbulent burst across a barrier. The top curve represents the mean velocity of the burst that experiences a significant slowing down within the barrier. In such a model, reducing the initial velocity of the burst leads to a regime where the burst is trapped in the barrier. Although such a model captures many outstanding properties of the interplay between the turbulent burst and the transport barriers, it is to be underlined that it does not include diffusive effects nor a self consistent evolution of the velocity field. Comparing this model to actual simulations, one finds that in both cases the squeezing effect reverses as the structure moves out of the barrier. Furthermore, when taking into account a residual cross-field diffusive transport, the increase of the perimeter enhances the diffusion process, hence reducing the contrast 
between the front pressure and the background pressure which in turn further reduces the front velocity. As a consequence the front can be trapped in the barrier and smoothed out by the residual diffusion. This mechanism is reminiscent of the Dupree mechanism recalled above with an amplification of the diffusive process by the shearing effect. In all these issues a crucial question is the exact nature of the transport controlled by the barrier as well as that of the remnant transport across the barrier [13].

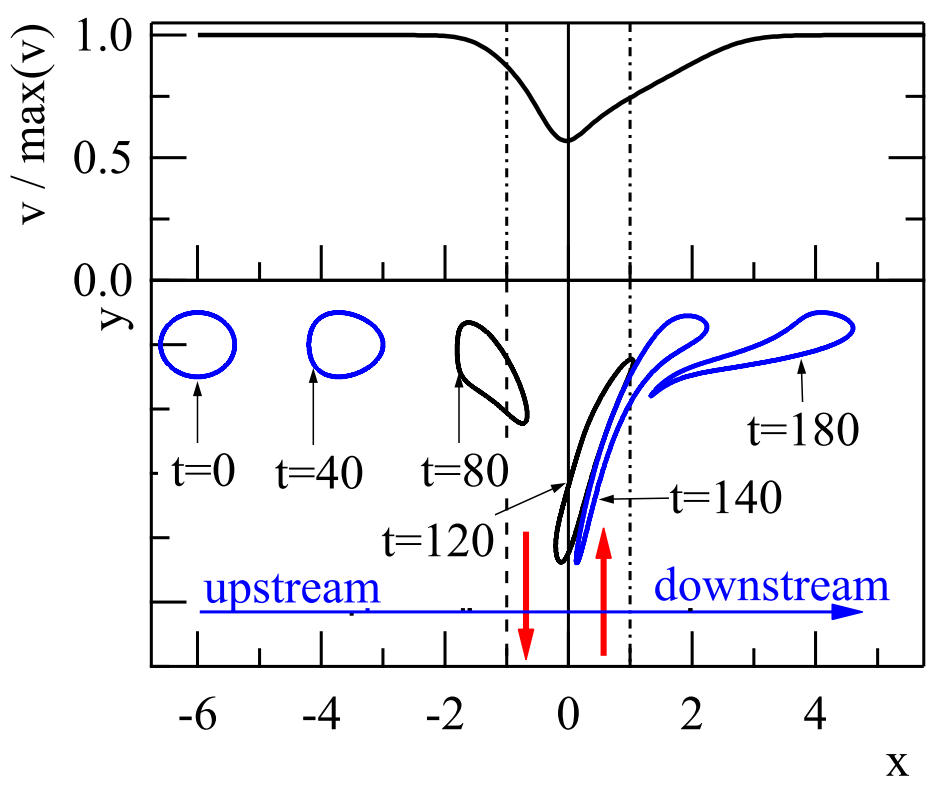

Figure 1: Lower panel: sketch of the propagation of a burst through a barrier. The transport direction is normalized to the width of the shearing region (gaussian shape). The burst location is given at various times, $t=0$ and $t=40$ upstream, in the barrier region, $t=80 t=120$ and $t=140$, and downstream $t=180$. The shearing directions is indicated by the vertical arrows. Top pannel: variation of the burst velocity during the propagation with a marked slowing down in the barrier region.

For the following, it is important to stress important properties of such a mechanism. First, the radial extent of the barrier cannot be directly compared to the size of the turbulent burst away from the barrier to estimate the stopping capability of the latter since the radial squeezing governed by the shear effect will completely modify the geometry of the burst. Second, the turbulent structure having a finite size, it can extend both in the barrier region where diffusion tends to strongly deplete the structure and in the vicinity of the barrier where the large gradients that build-up will strongly amplify the contrast between the pressure of the front and the background pressure. The balance between such conflicting mechanisms for a single pressure burst are difficult to predict. Depending on details of the barrier and of the turbulent burst, such non-local effects can yield either a further stabilization or a strong amplification of the structure as it moves out of the barrier region.

It is interesting to note that the coupling of turbulence to magnetic structures, such as Neoclassical Tearing Modes (NTM), leads to similar physics issues [36]. The spreading of turbulence within and across the magnetic island can then back-react on the NTM structure itself since the island width is governed by the transport process within the island, the latter then departing from the neoclassical paradigm. Furthermore, it then seems that such an island structure could exhibit a transmission factor departing from unity for the large bursts 
of turbulence thus modifying the turbulent transport properties downstream from the NTM.

Turbulence spreading into linearly stable regions has been investigated for ITG turbulence in global gyrokinetic codes [37]. It is characterized by a slowing down of the turbulence front within the linearly stable region. A similar setting is implemented in most global codes to stabilize turbulent transport via buffer regions located at the radial boundaries of the simulation region. These buffer regions are characterized by an artificially enhanced diffusion that quenches the turbulence leading to a spreading effect but without driving a barrier since the enhanced diffusive transport does not induce a significant change in the gradients [19]. These gyrokinetic simulations show that spreading effects within a transport barrier are generic of plasma turbulence but do not allow one to analyze turbulence burning through the transport barrier. More recently, gyrokinetic simulations in flux driven regimes have revealed a corrugated structure of the temperature profile that appeared to be governed by a quasi-regular radial pattern of zonal flows. The latter are sheared axi-symmetric flows generated by turbulence [38, 39, 40].

From the experimental point of view the interplay between self-organized transport events and transport barriers have been reported but associated to ELM relaxation events, hence on meso-scales. First, it is known that perturbations of the ETB can trigger ELMs. This is the case of sawtooth perturbations reaching the ETB and triggering an ELM as well as pellet perturbations that trigger ELMs and are thus contemplated as a means to monitor the ELM activity. A very interesting case of the erosion and inward radial displacement has been reported for an ITB perturbed by a sequence of type I ELMs. In this case, it has been shown that the series of fronts triggered by the ELM relaxation events propagate inward with a ballistic motion and interact with the ITB. The ITB response to each front is an inward shift together with a degradation of the barrier. This process ends as the ITB vanishes [41].

\subsection{The turbulence model of the simulations}

The stopping capability of transport barriers has been addressed in several papers, predominantly in fluid simulations where conditions for the onset of a transport barrier are easier to implement. We consider here barriers that are triggered by an external setting, either a mechanism that suppresses turbulence altogether within a prescribed region [27, 33, 34], or by driving a shearing region at the barrier location $[42,43,44]$. The role of these turbulent bursts in the quasi-periodic collapse of the edge transport barrier is still an open question [45]. We shall concentrate here on simulations with the TOKAM-2D code $[16,35]$. In these simulations, the TOKAM-2D model only addresses particle transport with given constant temperatures so that the energy of the coherent structures is proportional to the density. The model is that of interchange governed turbulence that exhibits in flux driven simulations significant intermittent transport events or turbulent bursts. The latter are characterized by ballistic propagation of overdense structures (downstream) as well as ballistic propagation of sub-dense structures (upstream). The model is considered in the flute approximation that assumes all quantities constant along the magnetic field lines. In this model, field lines connect two plasma facing components in the peripheral region of tokamak plasmas. This symmetry is therefore in line with the filamentary structure of the turbulent bursts. This allows one to reduce the model to two dimensions, the radial direction $x, x=(r-a) / \rho_{s}$ where $a$ is the plasma minor radius, $r$ the radius of the considered magnetic surface and $\rho_{s}$ the hybrid Larmor radius, and an angle that describes the poloidal angle on the magnetic surface $y$, $y=a \theta / \rho_{s}$ where $\theta$ is the actual poloidal angle. The angle coordinate is periodic so that the average of any particle flux on a given magnetic surface yields the radial component of the flux. Parallel loss terms to end plates govern a loss of particles and the relation between the parallel current and the electrostatic potential [33]. Our version of the TOKAM-2D model is therefore restricted to the SOL (Scrape-Off Layer) region, $x \geq 0$. However the rich physics that can be observed in these simulations provides a rather comprehensive physical setting for the analysis proposed in this paper based on the interplay of independent stochastic pro- 
cesses. The aim of this Section is to determine what aspects of the numerical simulations fall in line with the models proposed in Section 3 and to evaluate means to determine the characteristics of the stochastic processes and ultimately to test the analytical predictions. It is important to remark that the stochastic model also provides a guide line to determine key parameters that characterize the interplay between the so-called turbulent bursts and the transport barrier.

The equations that govern the model are the particle balance equation for the electron density $n$ and the charge conservation equation in the quasineutral asymptotic limit, which then restricts to $\operatorname{div}(\mathbf{j})=0, \mathbf{j}$ being the total current. The particle balance equation Eq.(1) includes the leading order particle flux due to the electric drift velocity, which takes the form of the Poisson bracket between the normalized electric potential $\phi$ and the density, together with a source term $S$, the parallel particle sink $n \sigma_{\|} \exp (\Lambda-\phi)$, and a small scale transverse diffusion smoothing term with coefficient $D_{\perp}$. It is to be noted that while the source term is localized radially, the loss terms is distributed in the simulation region and proportional to the density. The particle flux that drives the turbulence therefore decays as the radial distance from the source increases. For convenience and simplification of the simulations, higher order nonlinear terms are not taken into account. In these expressions, $\Lambda$ is the plasma potential with respect to the reference potential of the wall, it includes the plasma floating potential and the biasing potential where applied. The control parameter of the parallel particle loss term $\sigma_{\|}$is proportional to the normalized saturation current. The charge balance equation Eq.(2) takes the form of the evolution equation of the vorticity $W=\Delta_{\perp} \phi$, with an electric drift convection, the Poisson bracket term between the electric potential and the vorticity, together with a diffusive damping of the convective motion with diffusion coefficient $\nu_{\perp}$. Charge sources and sinks are due to the curvature drift, here simplified to a coupling to the poloidal density gradient, the coupling interchange term being taken constant equal to $g$. This $g$-term is comparable to the gravity term when addressing buoyancy effects in a neutral fluid. The specific charge loss terms due to the parallel current to the wall (second term on the right-hand side of Eq.(2)), governs the damping of the large scale structures.

$$
\begin{aligned}
\partial_{t} n+[\phi, n] & =D_{\perp} \Delta_{\perp} n-\sigma_{\|} n \exp (\Lambda-\phi)+S \\
\partial_{t} W+[\phi, W]+g \partial_{y} \ln (n) & =\nu_{\perp} \Delta_{\perp} W+\sigma_{\|}(1-\exp (\Lambda-\phi))
\end{aligned}
$$

In the absence of biasing, the system exhibits transport in bursts from the source $S$, which is localized radially, to the particle sink at the wall via the parallel loss term. The mean density profile converges towards an exponential profile typical of the SOL plasmas, the e-folding length $\lambda_{n}$ being determined by the balance between the characteristic turbulent transport time scale and the parallel loss term. For strong turbulent transport, $\lambda_{n}$ is large, and, conversely for a reduced transport, $\lambda_{n}$ is small leading to strong gradients. The linear instability analysis indicates that a positive growth rate is achieved when the drive term $-g \partial_{r} n / n$ exceeds a critical value that stems from the various damping mechanisms (the diffusion terms and the parallel loss terms).

In the TOKAM-2D code, barriers have been set either by modifying the $g$ term that governs the interchange instability [33], or by applying a biasing electric field with prescribed shape and magnitude $[42,43]$. In the model, the source term $S$ is located at a given radial location and turbulence is generated for the increasing values of $x$ with respect to the source position. With respect to the barrier, upstream refers to the region extending between the barrier and the source ( $x$ smaller than the value at the barrier, and conversely downstream is the region for $x$ larger than the barrier value. The former barrier with $g$ set to zero in a given radial region, directly modifies the linear growth rate of the unstable modes. It will be called a hard barrier since only the damping terms are effective within the barrier so that all linear eigenmodes are stable within the barrier region. The magnitude of the coherent structures that propagate within the barrier must then decay exponentially. The model based on 
the shearing effect of a biased electric potential is more complex insofar that the turbulence can generate a fluctuating electrostatic potential that competes with the external drive. The overall properties of the transport barrier then depend on the plasma response, such a barrier will be called a soft barrier. Another origin for the complexity of this model is the competing stabilizing mechanisms between the shearing effect (second derivative of the electric potential), and that governed by the third derivative [27]. The contribution of the plasma response to the electric potential and the lack of a predictive and quantitative model to describe the shearing stabilizing effect do not allow one to define precisely the region where turbulence is damped nor the effect of the damping mechanism on the magnitude of the turbulent bursts.

It is to be noted that in all simulations a parallel particle loss term is considered so that the amplitude of the pedestal will be set by a balance of the incoming energy via the intermittent transport due to the turbulent burst together with the given diffusive transport and the parallel loss term that increases linearly with the energy stored in the barrier. The balance between this loss term and the minimum diffusion coefficient leads to a maximum possible density gradient within the system determined by the characteristic scale $\lambda_{s}=\left(D_{\perp} / \sigma_{\|}\right)^{1 / 2}$. This bounds the available energy that could be expelled from the barrier. Another consequence is that the particle flux that drives the turbulent transport strongly decays at the barrier location so that the particle flux beyond the transport barrier is significantly reduced. These properties are specific of the barrier implemented in the SOL region and are a limitation both when comparing the present simulations to the stochastic model or when comparing the simulation to plasma transport barriers in the core plasma where there is no parallel loss terms to the wall $\left(\sigma_{\|}=0\right)$.

\subsection{Hard barrier case}

The clear-cut turbulence stabilizing mechanism imposed in the case of the hard barrier allows one to investigate the response of the barrier to incoming turbulent bursts. Our present analysis is twofold, on the one hand one can compare standard signatures that are used to characterize the barriers to the structure of the underlying stabilizing mechanism and on the other hand one can estimate the stopping capability of the barrier. We recall that the hard barrier is implemented by setting $g=0$ in a given radial domain of extent $\Delta_{B}$ with poloidal symmetry so that all modes are linearly stable.

A first issue when addressing the physics of transport barriers is to define the transport barrier itself. From an experimental point of view, this is often considered as a self explanatory concept. Most estimates of the barrier width are based on the increase of relevant gradient scales, for instance, when addressing the control issue of the Internal Transport Barrier a measure of the barrier strength was introduced [46] as the normalized ion temperature gradient. Let us introduce the total particle flux $\vec{\Gamma}_{\text {tot }}$ as the sum of the turbulent flux $\vec{\Gamma}_{\text {turb }}$ governed by the radial component of the electric drift velocity $\vec{v}_{E}=\vec{E} \times \vec{B} / B^{2}$, $\vec{\Gamma}_{t u r b}=n \vec{v}_{E}$, and of the underlying diffusive flux $\vec{\Gamma}_{d i f}=-D_{\perp} \vec{\nabla} n$. In order to reduce the fluctuation range, we consider the fluxes averaged on the poloidal angle so that $\Gamma_{\text {tot }}, \Gamma_{\text {turb }}$ and $\Gamma_{\text {dif }}$ are the radial component of the total, turbulent and diffusive fluxes averaged on the magnetic surfaces. The relevant criterion is then defined as the ratio $R_{B}$ between the poloidally averaged turbulent particle flux and the poloidally averaged total particle flux, $R_{B}=\Gamma_{\text {turb }} / \Gamma_{\text {total }}$. For a given total particle flux, $\Gamma_{t o t}=\Gamma_{\text {turb }}+\Gamma_{\text {dif }}$, one finds the two opposite limits: either the transport is dominated by the turbulent transport, and on average one finds $\Gamma_{\text {tot }} \approx \Gamma_{\text {turb }}$ so that $R_{B} \approx 1$, or the turbulence is quenched, so that $R_{B} \approx 0$ since $\Gamma_{\text {turb }} \ll \Gamma_{\text {tot }}$. In steady state $\Gamma_{\text {tot }}$ is close to the statistical mean value that comprises the particle source $S$ minus the parallel loss term of particles between the source location and the considered radial position. At a given time the decrease of the turbulent flux must then be compensated by an increase of the diffusive transport, and therefore of the density gradient $\nabla n$. As pointed out previously, this gradient will not exceed the value $\nabla^{*} n=\Gamma_{t o t} /\left(-D_{\perp}\right)$ 
that is reached when there is no turbulent transport. Since $\nabla n=\left(\Gamma_{\text {tot }}-\Gamma_{\text {turb }}\right) /\left(-D_{\perp}\right)$, one can readily show that $\nabla n / \nabla^{*} n=1-\Gamma_{\text {turb }} / \Gamma_{\text {tot }}$ and therefore $R_{B}=1-\nabla n / \nabla^{*} n$. Our criterion on the value of $R_{B}$ is directly linked to the value of the gradients and comparable therefore to the criteria used in the experimental analysis. It is to be noted that if the radial density gradient is positive, especially between successive turbulent bursts, then the diffusive flux is negative so that $\Gamma_{\text {tot }} \leq \Gamma_{\text {turb }}$ and therefore $R_{B} \geq 1$.

As reported in [33], the radial dependence of $\left\langle R_{B}\right\rangle$, the time average of $R_{B}$, is different from

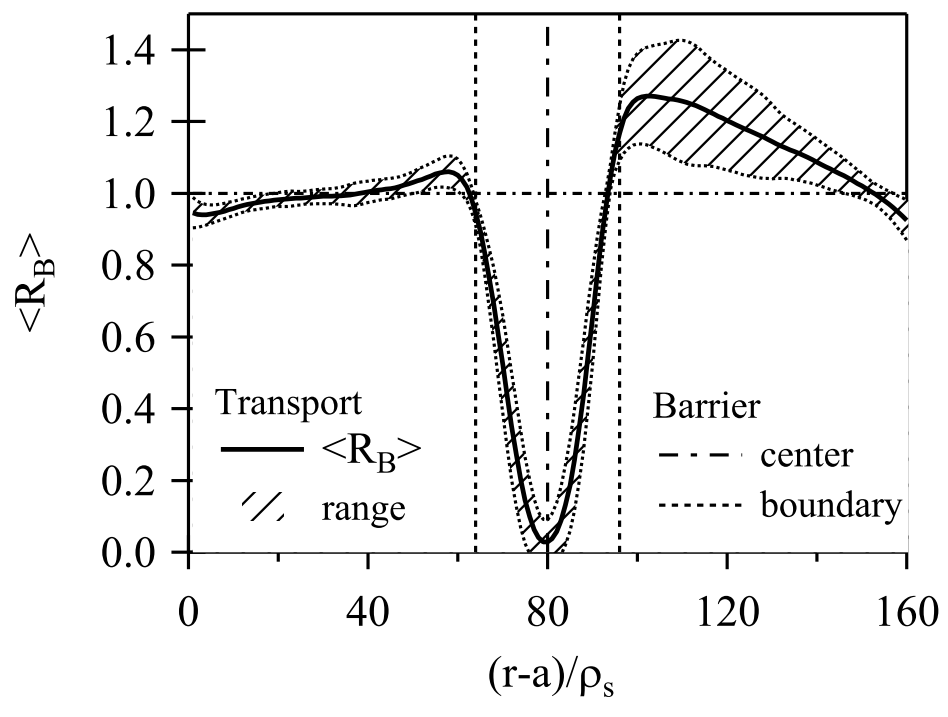

Figure 2: The bell shaped decrease of turbulent region within the stable region exemplified by the profile of the time averaged criterion $R_{B}$ and its fluctuations.

the square shape that characterizes the change in value of $g$, see figure 2 . One finds that $\left\langle R_{B}\right\rangle$ departs significantly from 1 outside the barrier region. It departs significantly from 1 within the barrier region set by $g=0$ where it exhibits a characteristic bell shape. In the present simulation, it does not vanish at its minimum, the latter being located in the center of the barrier. On both sides of the barrier one observes two areas where $\left\langle R_{B}\right\rangle$ exceeds unity. This indicates that the diffusive transport is opposite to the turbulent transport, and therefore that the density gradient is positive over significant periods of time. Such a feature is also observed in standard turbulent simulations [27] where the most probable value of the density gradient is positive. In these two regions this feature is emphasized so that even the mean density gradient is positive. In the downstream region, these aspects are particularly important since the system has not reached statistical equilibrium in these simulations. This also translates into large fluctuations of $R_{B}$. As the system evolves in time, a more symmetrical shape of these two wings appears to build-up. At the boundary of the $g=0$ region spreading of turbulent transport is observed. A weak dip of turbulent transport occurs prior to the stable region, while spreading of turbulent transport within the stable region is significant so that there is no region where $\Gamma_{\text {turb }}=0$ is satisfied at all times. From this point of view, one is then led to introduce a criterion to define the transport barrier. We thus define the points belonging to the effective barrier to be those for which $\Gamma_{t u r b} / \Gamma_{t o t} \leq 0.2$, namely those points where the average gradient increases by a factor larger than 4 assuming that in turbulent transport conditions $\left\langle\Gamma_{\text {turb }}\right\rangle /\left\langle\Gamma_{\text {tot }}\right\rangle \approx 0.8$. This criterion allows us to define a time and space dependent measure of the effective barrier location to investigate its statistical properties. As readily seen on figure 2, the barrier region is narrower that the region constrained by 
$g=0$.

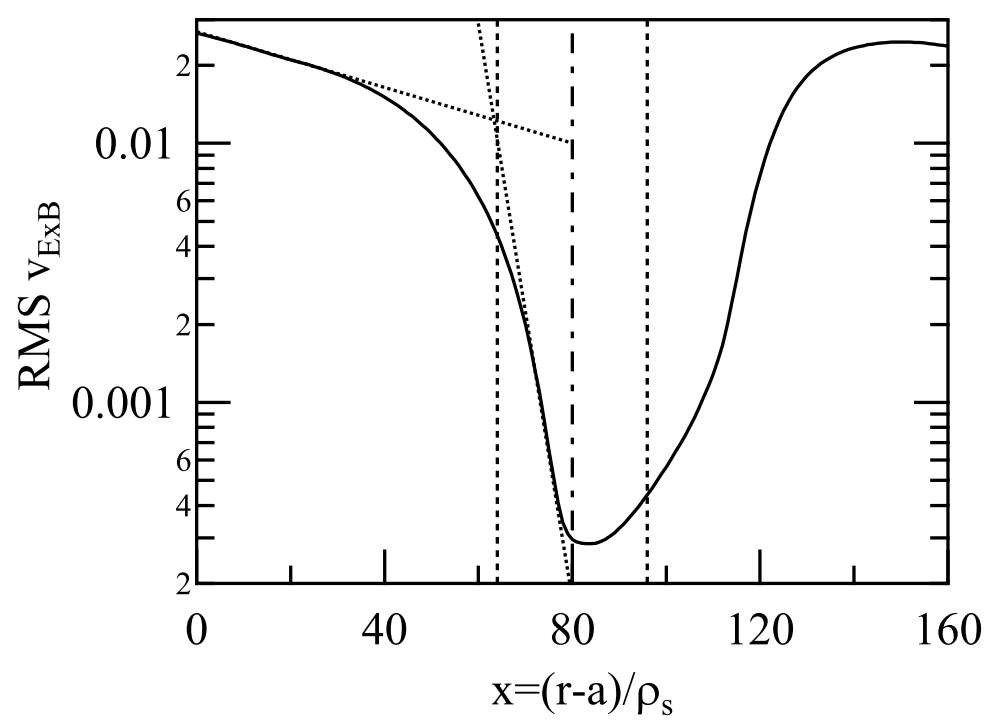

Figure 3: Variation of the root mean square value of the radial component of the $E \times B$ velocity considered as characteristic of the turbulent burst velocity across the barrier region.

We thus compare two aspects of the transport barriers. On the one hand, the region where turbulence is reduced is well defined in the case of hard barriers so that the width $\Delta_{B}$ of the region governed by turbulence reduction is prescribed. On the other hand when analyzing the transport properties one finds that the effective barrier exhibits significant fluctuations together with a reduced effective width. In the upstream region with respect to the barrier, the fluctuations of $R_{B}$ must be governed by the statistical properties of the PDF of the turbulent bursts in the turbulent region combined to the stopping capability of the barrier, namely the law that governs the decay of the turbulent burst magnitude within the barrier, see figure 3. A complete investigation of this point should be based on statistics of slowing down of the turbulence bursts based on the discrimination of the latter $[25,26]$ and analysis of the evolution of their pattern within the barrier. Such an analysis is very demanding and has not been carried out for these simulations. Rather, the behavior of the $E \times B$ drift velocity is investigated. Since its mean value is vanishing, one computes the Root Mean Square value of the radial projection of this velocity, see figure 3 . One also observes the spreading effect on this characteristic radial velocity of the turbulent bursts, with a dip prior to the stable region, a sharp exponential fall-off in the upstream side of the stable region and an increase in its downstream region, albeit with a much smaller slope. Since statistical equilibrium is not yet reached, it is difficult to assess the degree of asymmetry in the spreading properties between the downstream and upstream regions. Key properties that emerge from this analysis are a strong decay of the turbulent burst velocity into the barrier, typically by a factor 30 .

The symmetric shape of the bell shaped form of $R_{B}$, figure 2 as well as the increase of the radial velocity within the barrier must be governed by the spreading of holes, sub-dense turbulent bursts that propagate upstream, hence from the turbulent region on the downstream side of the barrier into the barrier. These holes are associated to electric dipoles that are comparable to those driving the "usual" overdense turbulent bursts but with opposite 
signs. The spreading within the barrier of the dipole structures due to holes can therefore generate outgoing turbulent bursts from the $g=0$ region, see figure 4 . In this snapshot at a given time, one can note that the turbulent burst that develop in the downstream region are connected to the barrier region as exemplified by the constant density contour lines. Furthermore, the density structures in the upstream part of the barrier have an opposite behavior when compared to that of the downstream density structures. A local density valley in the upstream region corresponding to the outgoing turbulent bursts in the downstream region. It seems that this example supports the interpretation of a turbulent burst spreading from the upstream region, hence driving a density depletion there, and reaching the barrier region from which the turbulent bursts are emitted, figure 4. This density transmission will be most significant when this event coincides with the spreading of a hole reaching the same region of the barrier. This then allows the decaying turbulence within the barrier to bridge the upstream and downstream region and provide turbulent transport across the barrier.

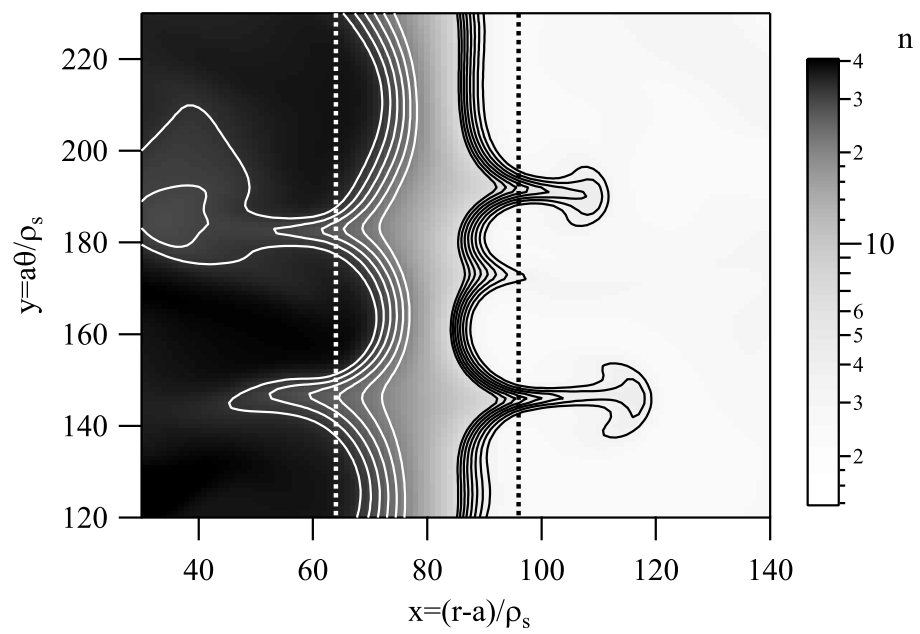

Figure 4: Spreading of coherent structures into the barrier domain.

Let us now analyze the decay mechanism of the turbulent burst radial velocity within the barrier. A strongly simplified linear analysis can be performed to exemplify this property in the vorticity equation. Since $g=0$ the vorticity equation for $W=\Delta_{\perp} \phi$ is decoupled from the density equation. Linearizing the vorticity convection term so that $[\phi, W]=v_{E} \partial_{r} W$ where $v_{E}$ is assumed to be constant, one then obtains $\omega=k_{r} v_{E}-i\left(\nu_{\perp} k^{2}+\sigma_{\|} / k^{2}\right)$. One thus recovers that the turbulent burst radial velocity is governed by $v_{E}$. The stabilizing effect appears in the turbulent burst velocity that decays exponentially in time with e-folding time $1 / \gamma$ and $\gamma=\nu_{\perp} k^{2}+\sigma_{\|} / k^{2}$. Large structures are damped by the parallel electric current via the the loss term $\sigma_{\|} / k^{2}$, while small scales are damped by the diffusion-induced damping $\nu_{\perp} k^{2}$. The intermediate regime such that $\nu_{\perp} k^{2} \approx \sigma_{\|} / k^{2}$ provides the smallest damping rate and determines the geometry of the structures that spread within the barrier, $k^{2}=\left(\sigma_{\|} / \nu_{\perp}\right)^{1 / 2}$ for wave-like structures. For a structure with a poloidal modulation with wave vector $k_{y}$ and radial exponential decay with rate $\lambda_{r}$, hence $k^{2}=k_{y}^{2}-\lambda_{r}^{2}$, one finds therefore $\lambda_{r}^{2}=k_{y}^{2}+\left(\sigma_{\|} / \nu_{\perp}\right)^{1 / 2}$. Since the wave vector $k_{y}$ is selected according to a similar rule, one then finds: $\lambda_{r}^{2}=2\left(\sigma_{\|} / \nu_{\perp}\right)^{1 / 2}$. The decaying solution exhibits a radial decay length that is a factor 2 larger than the poloidal wave length. As the turbulent burst propagates into the barrier, its velocity decreases exponentially so that a penetration length can be computed and is found to be $\Delta_{\text {turbulentburst }}=v_{E 0} / \gamma$, where $v_{E 0}$ is the turbulent burst velocity 
as it enters the hard barrier region and where $\gamma$ is the linear growth rate. For a given extent of the barrier, $\Delta_{B}$, one finds therefore that the barrier discriminates the incoming turbulent bursts according to their velocity: a density structure with a large radial velocity will spread across the barrier provided $v_{E 0}>\Delta_{B} \gamma$, while those with a radial velocity smaller than $\Delta_{B} \gamma$ will be stopped within the barrier. This strongly simplified calculation underlines three key features of the barriers, first that the stopping capability depends on the barrier width $\Delta_{B}$, second that this stopping capability also depends on the magnitude, or velocity, of the incoming density burst, third that the burst is not stopped at once as it reaches the barrier, but gradually decays. The hard barrier model is thus convenient since the prescribed stable region is straightforward to interpret. The behavior of the turbulent bursts in that region being deterministic the only stochastic processes at play are governed by the properties of the incoming turbulent bursts and holes that spread into the barrier from either sides of the barrier. However, one also finds that the response of the burst-barrier system is complicated and in particular that determining the stabilized region from the transport properties, such as the particle flux, is not readily achieved.

\subsection{Soft barrier case generated by external biasing}

In experiments, the occurrence of barriers is governed by a stabilizing mechanism that is generated by the plasma itself. While the exact model for the barrier build-up is still lacking, there is a broad consensus that eddy-shearing plays a major role [4]. We thus investigate these shearing barriers where external shearing is introduced to trigger the barrier [27, 44]. Although such a model will not recover the full degree of freedom governed by a fully selfconsistent barrier onset, it introduces important changes in the approach. First, the plasma response to the external drive can compete with the latter, either enforcing or reducing the stabilizing effect. One thus obtains such degree of self-consistency in the model. A second effect is that the actual stabilizing mechanism is not known so that no criterion is available a priori to determine the region where turbulence is actually stabilized, nor can one assess the degree of stabilization that is achieved. The plasma response adds a further stochastic process to the analysis of the interplay between the turbulent bursts and the barriers, namely the barrier width as introduced in the models, Section 3. We are also led to analyze the barrier solely in terms of criteria such as the ratio of the turbulent transport over the total transport, $R_{B}$, as introduced in the previous Section 2.3 for the hard barrier.

In this Section, we consider numerical simulations in the case of a barrier triggered by polarizing the SOL plasma [43]. As in the case of the hard barrier, this model of a shearing barrier exhibits parallel losses to the wall which directly leads to a saturation of the energy build-up in the barrier, and therefore of the pedestal height. Furthermore, in this soft barrier case, the system appears to remain with a gradient much smaller than would be required to reach the state characterized by barrier relaxation modes [45]. Such events are not readily observable in these simulations.

The biasing potential used in the simulation has a Gaussian profile, peaking at $\left(r_{g}-\right.$ a) $/ \rho_{s}=92$ with characteristic width $\Delta_{g} / \rho_{s}=16$. The biasing electric potential is then proportional to $\exp \left(-\left(r-r_{g}\right)^{2} / \Delta_{g}^{2}\right)$. Consequently, the maximum shearing rate of the electric field then exhibits three peaks, the main one at the maximum of the Gaussian and two secondary peaks located at $r_{s}=r_{g} \pm \sqrt{3 / 2} \Delta_{g},\left(r_{s}-r_{g}\right) / \rho_{s} \approx 11.3$. In figure 5 , we have used the ratio $R_{B}$, introduced for the hard barrier case, as a criterion to identifying the barrier region. The top panel of figure 5 presents the time trace of the radial profile of $R_{B}$. The dark regions, within the contour lines $R_{B} \leq 0.4$ correspond to regions of strongly reduced turbulent transport. A rather complex pattern can be observed with patches of reduced transport localized in the vicinity of radius $(r-a) / \rho_{s} \sim 90$. These patches can extend either inward or outward from the $(r-a) / \rho_{s} \sim 90$ line, or can be rather symmetric. One can 

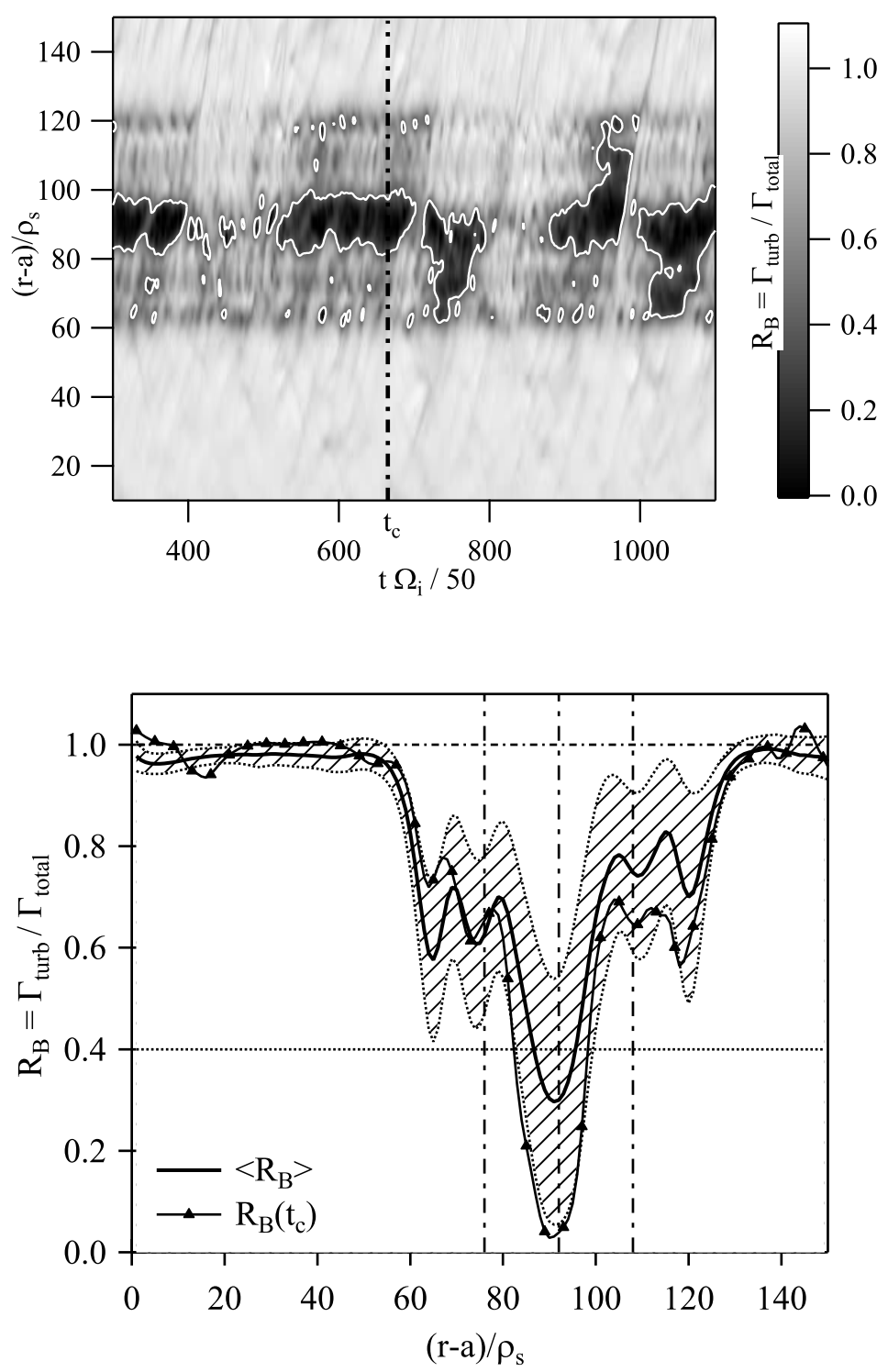

Figure 5: Top panel: 2D plot of $R_{B}$ values in the time-radius plane. The dark region corresponds to a strong reduction of the turbulent transport, $R_{B} \leq 0.4$, conversely the light gray and white region is dominated by turbulent transport, $R_{B} \sim 1$. The white contour plots correspond to the $R_{B}=0.4$ contour line. Bottom panel: time averaged value of $R_{B}$ and root mean square of the $R_{B}$ fluctuations, the dashed region around $\left\langle R_{B}\right\rangle$. The black triangle profile is the radial profile of $R_{B}$ at time $T_{m} \Omega_{i} / 50=665$. The vertical lines indicate the region where biasing control is applied, the center and lines at 2 times the width of the Gaussian shape.

also observe collapses of these strongly reduced turbulent transport regions. In particular, one notices a collapse of the patch prior to time $t \Omega_{i} / 50 \sim 1000$ that follows the trace of a ballistic event propagating with a velocity of the order of $2.5 \%$ of the sound velocity $c_{s}=\rho_{s} \Omega_{i}$. One also notices smaller patches with $R_{B} \leq 0.4$ that appear to cluster along the radial lines $(r-a) / \rho_{s} \sim 65$ and $(r-a) / \rho_{s} \sim 120$, rather symmetrically with respect 
to the $(r-a) / \rho_{s}=90$ line. The region affected by the reduction of turbulent transport extends typically from $(r-a) / \rho_{s}=60$ to $(r-a) / \rho_{s}=125$. One recovers these features when analyzing the time averaged profile of $R_{B}$ together with its fluctuations, lower panel of figure 5. The shape of $\left\langle R_{B}\right\rangle$ is similar to that of the second derivative of the biasing potential, hence to that of the electric field shear. One observes a main peak with a strong decay of the turbulent transport and two wings, quasi-symmetric, each exhibiting two secondary peaks. While the first peak can be compared to that of the imposed electric field shear, the second peak does not correspond to peaks of higher order derivatives of the biasing electric field. This feature as well as the width of the region where turbulent transport is modified is typically twice the characteristic width of the polarization profile. This strongly suggests that the plasma response plays a role in the effective shearing process. This aspect is also backed by the large fluctuations of $R_{B}$ whithin the region with reduced turbulent transport, unlike the hard barrier where the barrier width is set and where the fluctuations of $R_{B}$ within the barrier are comparable to that in the turbulent region upstream from the barrier, figure 2 .

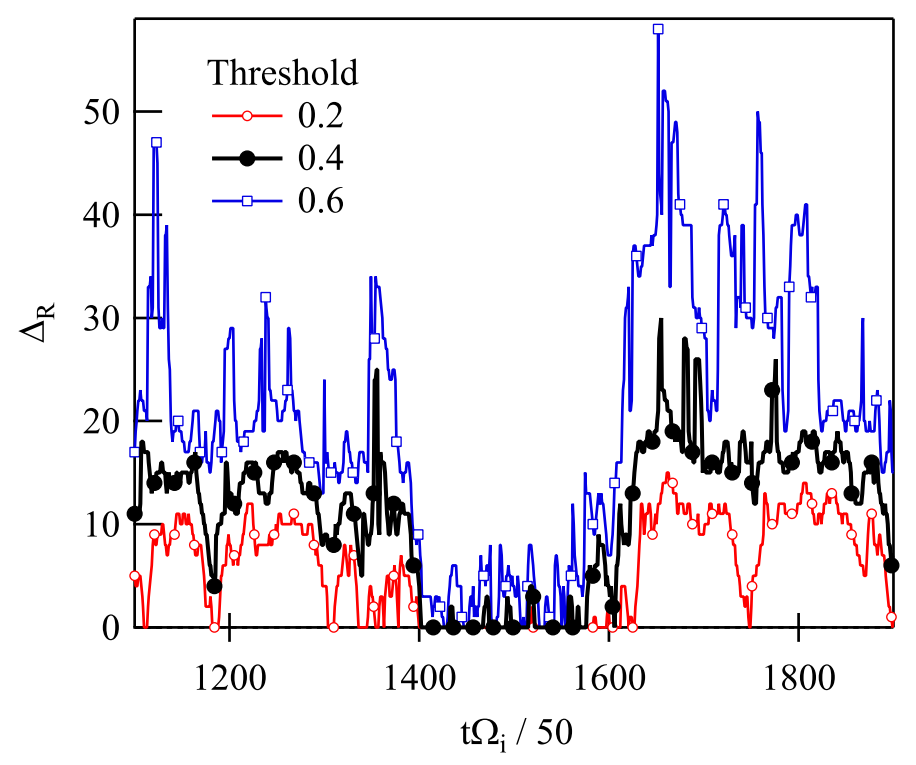

Figure 6: Time evolution of the radial extent of the transport barrier for three different threshold values of $R_{B}=\Gamma_{\text {turb }} / \Gamma_{\text {tot }}$.

We define the barrier width by a threshold value for $R_{B}$, the barrier extending in the region where $R_{B}$ is smaller than the threshold, figure 6 . Depending on the threshold value, the barrier width $\Delta_{R}$ dynamics is different although one can identify common trends, such as the barrier collapses and recovery. In fact small threshold values mean a strong reduction of turbulent transport with respect to the total one. In this case, the barrier is essentially composed by the parabolic shape located in the central region, around $(r-a) / \rho_{s}=92$, and its fluctuations are relatively small. For larger threshold values, the two peripheral regions around the central are then considered as part of the barrier. In these regions the plasma response produces a shear of the potential of the same magnitude than the one imposed by the external biasing term. The observed strong fluctuations in the reduction of turbulent transport, that is in the $R_{B}$ values, are the combined effect of fluctuations of the width of the stabilizing region as well as possible variations of the magnitude of the stabilizing mechanism. In what follows we decide to consider the barrier as the region where $\Gamma_{t u r b} / \Gamma_{t o t} \leq 0.4$. The 
corresponding Number Distribution Function of the radial extent is shown in figure 7. The integral of the NDF is the total number of points in the statistics. It is therefore proportional to the PDF and provides some insight into the statistics and in particular the statistical errors. We remark that the most probable event corresponds to the collapse of the barrier, that is null radial extent. Moreover, an almost constant probability is measured for radial extents varying from $1 \rho_{s}$ to $20 \rho_{s}$. Larger barrier extents are also observed but with typically a factor 10 reduction in frequency. The barrier extent is thus characterized by a mean width of the order of $9 \rho_{s}$ and a root mean square of its fluctuations of $7 \rho_{s}$, comparable to the mean value. This property reflects the strong fluctuations of the barrier size observed in figure 6 that support the description of the barrier width as a stochastic function.

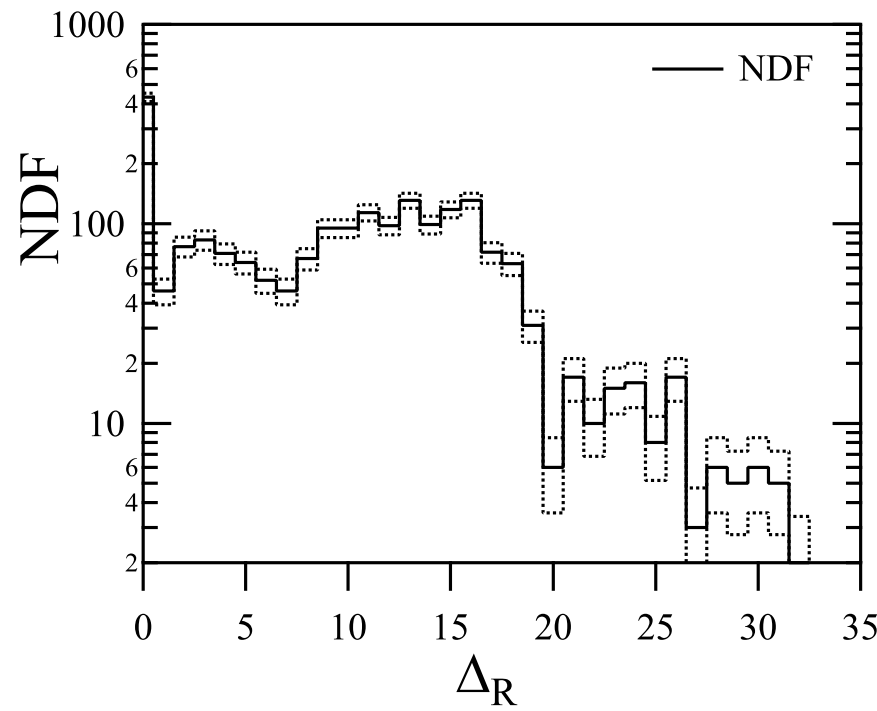

Figure 7: NDF of the radial extent of the barrier for $R_{B} \leq 0.4$. The dotted histograms are the NDF value $\pm \sqrt{N D F}$. They provide a statistical estimate of the error bars.

\section{Stochastic model of turbulence bursts and transport barriers}

We want to model the radial motion of coherent transport structures that are referred to as turbulent bursts or filaments through a transport barrier whose position fluctuates in time. We therefore introduce an interval $[0, L]$ representing the radial extent of an edge region including the pedestal and transport barrier. We consider the radial extent of the transport barrier to be a random variable $\Delta_{B}$, taking values in the interval $[0, L]$.

The key effect of the transport barrier on entering turbulent bursts is to slow them down and, for most of the incoming turbulent bursts, to stop them within the barrier (some explanations on this expected behavior can be found in e.g. reference [34]). However, it is possible that some events burn through the transport barrier. These events then determine the effective transport rate through the barrier, hence governing the pedestal height. Furthermore, they can also contribute to the relaxation of the transport barrier. In our approach, we then distinguish the turbulent bursts that succeed in crossing the transport barrier, which we call the escaping turbulent bursts, and the remaining ones, which we call the trapped turbulent bursts. The second quantity, after $\Delta_{B}$, that will be relevant for the model is precisely the 
spreading width, or stopping distance, of a turbulent burst inside the barrier. We will call this distance $\delta_{t b}$, and since it fluctuates from one turbulent burst to the other, we will also take it to be a random variable, independent from $\Delta_{B}$, and taking values in the same interval $[0, L]$. Trapped turbulent bursts will be those for which $\delta_{t b} \leq \Delta_{B}$, whereas escaping turbulent bursts are such that $\delta_{t b}>\Delta_{B}$.

The physical quantity we are interested in is the amplitude of the turbulent bursts that make their way out of the transport barrier, despite its slowing effect. In particular, we would like to see under which conditions the amplitude of the escaping turbulent bursts may take very large values with relatively large probability, which would correspond to an intermittent behavior of the time evolution of the turbulent burst amplitude. This calculation allows one to compute the tail of the PDF of the turbulent burst downstream from the barrier. Such predictions can thus be used to interpret the PDFs obtained either in simulations or actual experiments.

The last ingredient of our model is then the physical amplification mechanism through which escaping turbulent bursts can acquire "energy" as they move out of the transport barrier. We shall consider two different versions of the model. They differ in the mechanism generating the large values of escaping energy: in version $(T A)$ or "take away", the amplification predominantly takes place within the transport barrier, whereas in version $(C G)$, or "cross and grow", the amplification takes place in the turbulent region just outside the barrier. Of course these two mechanisms, that we treat separately as two limit cases, could both be present in a physical situation. The possible mechanism by which turbulent bursts can gain energy when escaping transport barriers corresponds to barrier erosion, i.e. the fact that part of the plasma energy stored inside the transport barrier is released through the escaping turbulent burst. The barrier then undergoes a micro-relaxation governed by the existence of the escaping turbulent burst. It is important to underline that a direct consequence of such a description is that the tighter the barrier, the more the relaxation event will behave like a meso-relaxation event [45]. As far as the second mechanism "cross and grow" is concerned, it is simply a case where the turbulent burst recovers standard conditions after having experienced the stabilizing mechanism within the barrier.

\subsection{Stochastic model $(T A)$ : "take away"}

We suppose that the significant amplification mechanism is due to a "pumping of amplitude" from the local environment inside the transport barrier. In particular, we suppose that the energy carried outside the barrier by an escaping turbulent burst is proportional to the number $N_{i n}$ of turbulent bursts having stopped and released their energy inside the barrier before the $N_{\text {in }}+1$ th turbulent burst escapes. The escaping turbulent bursts then govern part of the relaxation mechanism of the barrier allowing it to reach a statistical steady state. To investigate the stochastic process we analyze the Probability Distribution Function (PDF) of the amplitude of the outgoing turbulent bursts, and especially the behavior of the tail of the PDF for the large values of the amplitude, hence the scaling of the PDF at large amplitude.

Let $\mathcal{P}_{N}$ be the PDF that characterizes the fact that $N_{i n}$ consecutive incoming turbulent bursts have been trapped in the barrier while the $N_{i n}+1$ th turbulent burst is an escaping one. Given our working assumption regarding the energy release, the PDF of the outgoing energy will also be $\mathcal{P}_{N}$. The situation where the bursts of outgoing energy display an intermittent behavior corresponds to the case such that $\mathcal{P}_{N}$ has heavy tails (slow decay), i.e., the case where the probability of having a large number $N_{i n}$ of turbulent bursts trapped before one escapes is relatively large. This corresponds to a strong transport barrier. In this case, one also finds that the barrier acts as a filter, suppressing the smaller turbulent bursts and amplifying the escaping bursts.

We thus consider a large number of turbulent bursts, independent one another, that propagate with a ballistic motion towards a barrier of radial extent $\Delta_{B}$. To take into account the stabilizing mechanism, we assume that the barrier acts by slowing down the incoming turbulent bursts up to the point of stopping them. Let $\delta_{t b}$ be the distance required by the 
stabilizing mechanism to bring the velocity of the turbulent burst to zero. Let us further assume that this distance characterizes the turbulent suppression mechanisms and the intrinsic properties of the incoming turbulent burst but does not depend on the width of the barrier $\Delta_{B}$. The two random processes that govern $\delta_{t b}$ and $\Delta_{B}$ are then considered to be independent. Their realizations are characterized by their PDFs $\mathcal{P}_{\Delta}\left(\Delta_{B}\right)$ for the barrier width and $\mathcal{P}_{\delta}\left(\delta_{t b}\right)$ for the stopping distance.

The rule that determines if a turbulent burst is trapped or escaping is then readily obtained by comparing $\delta_{t b}$ and $\Delta_{B}$ with $\delta_{t b} \leq \Delta_{B}$ for a trapped turbulent burst and $\delta_{t b}>\Delta_{B}$ for an escaping turbulent burst. The possible resulting behaviors of $\mathcal{P}_{N}\left(N_{i n}\right)$ (where $N_{i n}$ is the number of consecutive occurrences of $\delta_{t b} \leq \Delta_{B}$ ) are particularly rich [47] if we allow the two variables $\delta_{t b}$ and $\Delta_{B}$ to vary with different time scales. The latter assumption can be regarded as well justified from a physical point of view since $\delta_{t b}$ is characteristic of a single turbulent burst while $\Delta_{B}$ characterizes a poloidally extended structure and may therefore vary on a longer time scale. To model this fact, we introduce another parameter $\eta \in[0,1]$. As $\eta$ approaches 1 the time scale of the two mechanisms will diverge. This parameter rules the variation of the barrier width $\Delta_{B}$ when a burst is trapped. It defines the probability that the barrier width is not changed by the trapping of a burst, a random event referred to as the resting case, while, with a probability $(1-\eta)$, it will be chosen according to its PDF $\mathcal{P}_{\Delta}\left(\Delta_{B}\right)$, an occurrence that we call the modified case. In our model at each time step we compare $\delta_{t b}$ and $\Delta_{B}$ after the random value of the resting-modified instance is ruled. To complete the rule that governs the barrier width, we assume that each escaping turbulent burst triggers a barrier readjustment in agreement with the idea that it corresponds to a micro-relaxation event. Consequently the width $\Delta_{B}$ is reset according to the $\operatorname{PDF} \mathcal{P}_{\Delta}\left(\Delta_{B}\right)$ with probability 1 after each such event.

The probability distribution $\mathcal{P}_{N}\left(N_{i n}\right)$ of $N_{i n}$ has been computed recursively [47], starting from $N_{i n}=0$ and increasing $N_{i n}, N_{i n}=1,2, \ldots$. The calculation is organized around the fact that each turbulent burst crossing the barrier can either be trapped, event labeled by $T$ or escape, event labeled by $E$. The trapped $T$ and the escaped $E$ events are of two kinds, the modified cases that follow a change of the barrier width, hence that exhibit a synchrony between the burst and the barrier width fluctuation labeled by $T_{m}$ or $E_{m}$ and the resting cases that do not follow such a change and are labeled $T_{r}$ or $E_{r}$. A sequence of events leading to an energy release is therefore represented by a chain of $N_{i n}$ characters $T_{m} T_{r} T_{m} \ldots T_{m} T_{r}$ followed by an ending character that is always $E_{r}$ or $E_{m}$. On average one expects that $\eta N_{i n}$ characters will be of resting events while $(1-\eta) N_{\text {in }}$ are modified events. For each chain of characters one can then compute its probability. More details are found in [47], and the asymptotic behavior of particular interest to evaluate the barrier is given in Appendix A.

Depending on the choice of the probability $\eta$, and of the PDFs $\mathcal{P}_{\Delta}$ and $\mathcal{P}_{\delta}$, one can prove in some cases that the PDF of the escaping turbulent burst, $\mathcal{P}_{N}\left(N_{i n}\right)$, has an algebraic tail, and, in other cases one can prove that this PDF has an exponential tail. The former corresponds to heavy tails and characterizes a tight barrier, while the latter will characterize leaky barriers. The results can be summarized as follows:

- in the case $\eta=0$, when almost surely each burst triggers a readjustment of the barrier width, $\mathcal{P}_{N}\left(N_{i n}\right)$ decays exponentially for all choices of PDFs $\mathcal{P}_{\delta}$, and $\mathcal{P}_{\Delta}$;

- in the case $\eta=1$, when almost surely only the micro-relaxation event triggers a change of the barrier width, there are classes of PDFs $\mathcal{P}_{\delta}$ and $\mathcal{P}_{\Delta}$ that allow one to obtain any possible (normalizable) power law decay of the distribution $\mathcal{P}_{N}\left(N_{i n}\right)$, namely: for $\mathcal{P}_{\delta}\left(\delta_{t b}\right) \propto \delta_{t b}^{\alpha}(\alpha>-1), \mathcal{P}_{\Delta}\left(\Delta_{B}\right) \propto\left(L-\Delta_{B}\right)^{\beta} \quad(\beta>-1), \mathcal{P}_{N}\left(N_{i n}\right)$ asymptotically scales like $N_{i n}^{-(2+\beta)}$ for large $N_{i n}$;

- for other values of $\eta, 0<\eta<1$, the decay is two-fold: the asymptotic behavior (large $N_{i n}$ ) is always exponential, while the decay for short and intermediate times depends on $\mathcal{P}_{\delta}$ and $\mathcal{P}_{\Delta}$. In particular, when $\mathcal{P}_{\delta}, \mathcal{P}_{\Delta}$ are constant (same probability for all possible values of $\Delta_{B}$ and $\delta_{t b}$, and when $\eta$ is close to 1 the exponential regime is only reached 
on times $\left(N_{i n}\right)$ of order $(1-\eta)^{-1}$, while for intermediate but large times, the decay is polynomial.

The case $\eta$ close to 1 thus yields a comparatively large probability to observe a relaxation event with a large energy. This energy is a signature of the large number of turbulent bursts trapped in the barrier without modifying its properties. These algebraic tails thus characterize tight barriers. As for earthquakes, there is then always a significant probability to experience a meso-relaxation mechanism, comparable to an ELM [45], or worse a macrorelaxation event that can trigger the loss of the ETB altogether. Polynomial distributions of the kind will exhibit a divergence of some moments of the distribution. In the nonlinear community such heavy tails are often taken as a definition of intermittency [48, 49, 50, 51]. It is to be noted that in such a regime one can readily expect the downstream turbulent bursts to be less frequent but with larger magnitude than the upstream ones, hence easier to identify. This situation is reminiscent of the "blob-intermittency" reported in the SOL [18]. Conversely, the case $\eta$ close to 0 with its exponential tail corresponds to a leaky barrier. Such a PDF is also reported for the turbulent flux without a barrier [27]. The other values of $\eta$ indicate that rather rich dynamics of the barriers can be expected on the basis of this model, the asymptotically exponential PDFs indicating a less problematic behavior on macro-time scales than would be expected on shorter times from the algebraic tail.

\subsection{Stochastic model $(C G)$ : "cross and grow"}

In this approach one considers that the turbulent burst is submitted to two opposite effects. On the one hand the barrier tends to stabilize it while on the other hand one assumes there is a mechanism that enhances its magnitude. For convenience, one can assign to each mechanism a different region in space. In the second region, one can assume that the turbulent burst extends over both the barrier region and the downstream region and can exhibit an amplification due to the fact that it connects the high energy pedestal to the foot of the pedestal, hence experiencing a very strong contrast with the background plasma. Such a double-layer barrier can be illustrated by the trajectory of a particle undergoing an increase of the potential energy (barrier layer) followed by a decrease of this potential energy as drawn on figure 8. Alternatively, one can view such a mechanism to be governed by two different time scales rather than being split in space. The evolution would then be opposite to the Dupree case, with a linearly stable evolution (governed by the barrier effect) followed by a non-linearly unstable evolution.

For the sake of convenience, let us consider a double layer barrier. The amplitude of each escaping turbulent burst propagating in the region downstream from the barrier is then assumed to be determined by two characteristic times:

- the time $t_{c}$ spent crossing the barrier during which the initial amplitude is gradually reduced;

- the time $t_{g}$ spent in the second region during which the amplitude increases.

The amplitude $A_{\text {out }}$ of the turbulent burst when leaving the barrier region (the quantity whose distribution we want to compute) can be written as a function of the turbulent burst amplitude prior to the barrier $A_{i n}$ as follows:

$$
A_{\text {out }}=A_{\text {in }} f_{c}\left(t_{c}\right) f_{g}\left(t_{g}\right)
$$

where $f_{c}$ is a decreasing function of its argument $t_{c}$, the crossing time of the stabilizing region, and, $f_{g}$ is a growing function of $t_{g}$, the relevant time during which the amplitude of the escaping turbulent burst increases.

The times $t_{c}$ and $t_{g}$ depend on the velocity profile $v$ of the turbulent bursts, which varies from one turbulent burst to the other. They also depend on the size of the barrier, which, as in the previous model, also fluctuates. The amplitude $A_{\text {out }}$ is thus a function of many 


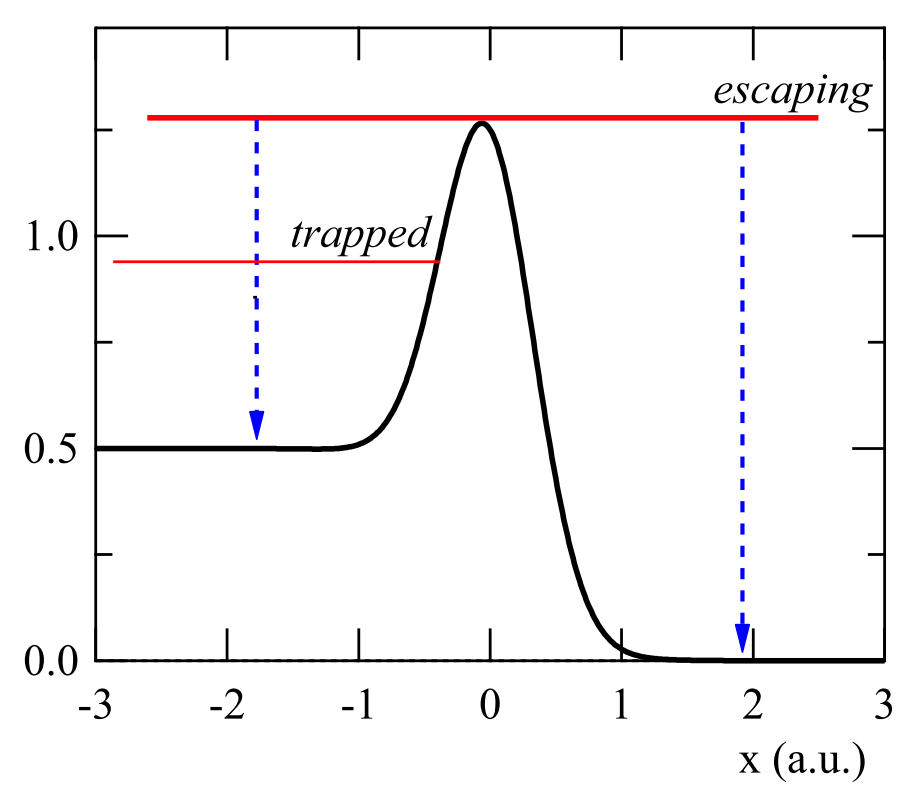

Figure 8: Model Cross \& Grow (CG): analogy with a potential barrier, when the energy of the incoming burst is smaller than the barrier potential the burst is trapped while the escaping burst has an increased energy due to the drop of the potential between the upstream and downstream sides.

random variables. We will concentrate on the dependence on the random variable related to the mechanism generating a large growth of $A_{\text {out }}$.

Let $x$ be the mean propagation direction of the turbulent bursts. As far as the velocity profile $v(x)$ is concerned, we suppose that a turbulent burst moves with constant speed $v_{t b}$ in the regions external to the barrier, and that its velocity decreases inside the barrier. With this assumption, we introduce in fact a decoupling between the amplitude of the turbulent burst, hence its "energy" content, and its propagation velocity within the barrier region.

As in model $(T A)$, we will denote by $\delta_{t b}$ the stopping distance of the turbulent burst inside the barrier. Thus, a turbulent burst is trapped if its velocity vanishes so that the time governing the movement of the turbulent burst amplitude tends to infinity. We further consider that $v_{t b}$ and $\delta_{t b}$ are random variables, in the sense that they fluctuate from one turbulent burst to the other. $\Delta_{c}$ and $\Delta_{g}$ denote respectively the width of the stabilizing region and that of the amplification region. If the turbulent burst velocity goes to zero inside the stabilizing region, i.e., if $\delta_{t b} \leq \Delta_{c}$, then the turbulent burst is trapped as in model $(T A)$. Conversely, if $\delta_{t b}>\Delta_{c}$, we assume that the turbulent burst will leave the barrier with the velocity it had at $\Delta_{c}$. For tractable analytical solution, we also assume that it will keep the same velocity during the time it experiences the subsequent growth. In other words, we suppose that the velocity profile $v(x)$ of the turbulent bursts is of the type depicted in figures 9 and 10.

The essential mechanism for the growth of the final amplitude is related to the fact that the amplitude of the barrier $\Delta_{c}$ can be arbitrarily close to the spreading distance $\delta_{t b}$ : this implies that the turbulent bursts leaving the barrier can have arbitrarily small final velocities, i.e., arbitrarily large growth of their magnitude. (Note that the analytical expressions still hold whenever the velocity of the turbulent burst in region "g" is proportional to the velocity at point $\Delta_{c}$, but not necessarily constant.) 


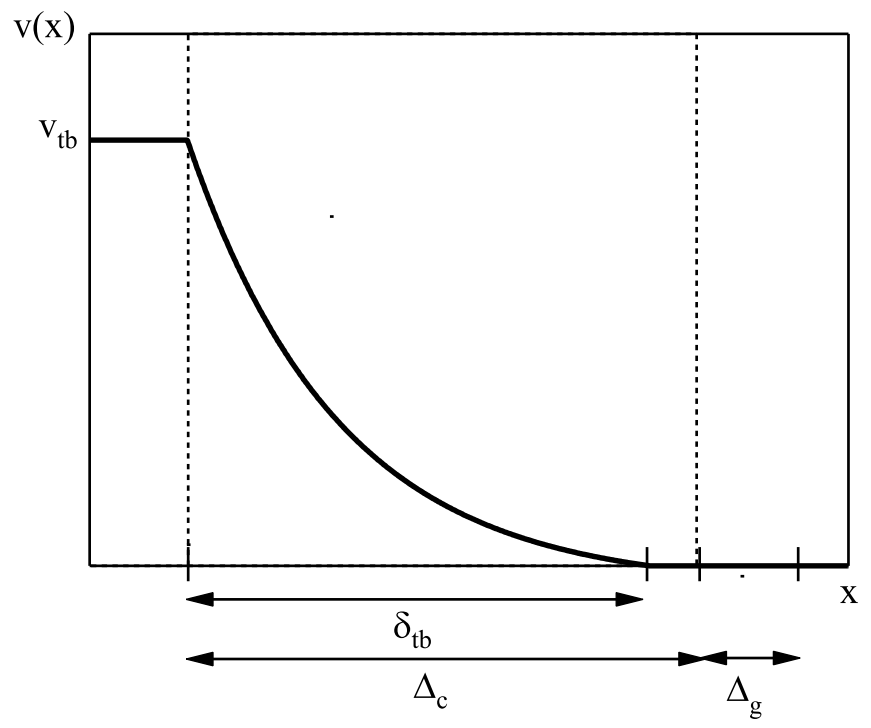

Figure 9: Model (CG): Case $\delta_{t b} \leq \Delta_{c}$ : the turbulent burst stops in the barrier.

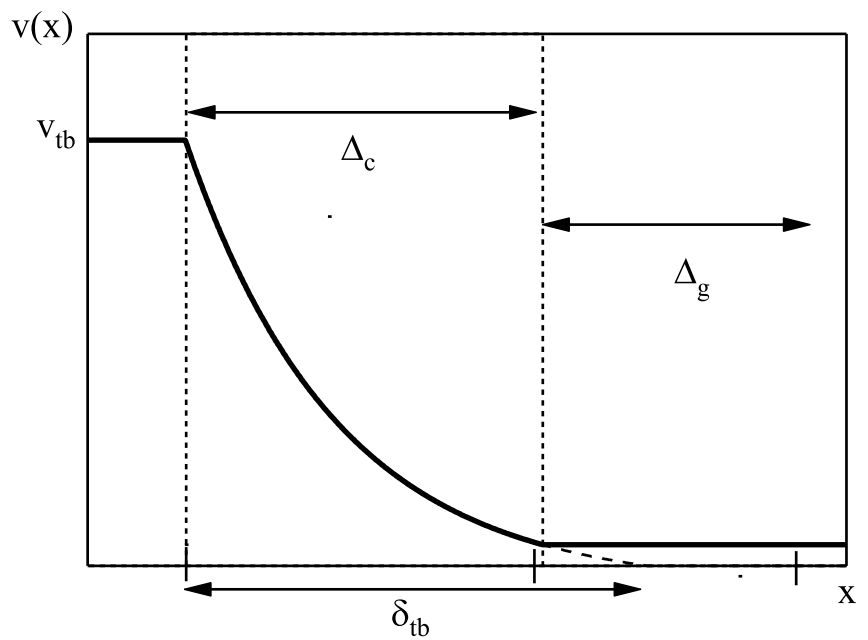

Figure 10: Model (CG): Case $\delta_{t b}>\Delta_{c}$ : the turbulent burst burns through the barrier.

The calculation performed in Appendix B leads to the following dependence of the amplitude $A_{\text {out }}$ on the normalized distance $z=\left(\delta_{t b}-\Delta_{c}\right) / \delta_{t b}$ between the spreading distance of the turbulent burst $\delta_{t b}$ and the width of the stabilizing region $\Delta_{c}$ :

$$
A_{\text {out }}(z) \simeq A_{\text {in }} f_{c}\left(\ln \left(\frac{1}{z}\right)\right) f_{g}\left(\frac{1}{z}\right)
$$

The form of $f_{c}, f_{g}$ is precisely what one should estimate when applying this version of the model to experimental or numerical data. In general, the situation where $A_{\text {out }}$ takes arbitrarily large values with large probability corresponds to the case where the growth of 
$f_{g}(1 / z)$ dominates the decay of $f_{c}(\ln (1 / z))$ as $z$ tends to zero. In Appendix B, we discuss the following cases:

- $f_{c}$ exponentially decreasing, $f_{g}$ exponentially increasing;

- $f_{c}$ exponentially decreasing, $f_{g}$ increasing as a power law.

For these cases, the PDF of $A_{\text {out }} / A_{\text {in }}$ has a power-law type decay, with diverging moments. Such a slow decay means that high values of the final amplitude of escaping turbulent bursts occur with large probability.

A key of the model is that we address the physics of coherent objects such that zero velocity is achieved as the structure can no longer be discerned. The leading property of the computed ratio $A_{\text {out }} / A_{\text {in }}$ is that the asymptotic behavior when $z \rightarrow 0^{+}$governs a logarithmic divergence of the crossing time, $T_{m} \propto \ln (1 / z)$, while the subsequent growth is governed by a larger divergence of its characteristic time since $t_{g} \propto 1 / z$. The model can be extended to situations such that $T_{m} / t_{g} \rightarrow 0$ when $z \rightarrow 0^{+}$. However, analytical estimates of the PDF of large amplitude turbulent bursts that have burned through the barrier can only be obtained for a restricted set of parameters.

The filtering aspect induced by the dynamics of such a barrier leads to three well defined behaviors. First, the turbulent bursts with small incoming velocity are trapped in the barrier. Second, the turbulent bursts with large incoming velocity experience a net decrease of their amplitude, since the growth time $t_{g}$ is not large enough given the large velocity still achieved at point $\Delta_{c}$. There are thus values of the incoming velocity such that the growth in the "g" region does not compensate for the decay inside the barrier. Third, the intermediate velocity turbulent bursts for which the penetration depth is comparable to but larger than the width of the stabilization region, $z \geq 0$. These latter turbulent bursts exhibit the largest amplification of their amplitude.

The CG model can appear to be rather artificial from the point of view of the standard picture of vortex shearing. However, when considering non-linear structures, there are cases such that this mechanism can be regarded as describing the appropriate asymptotic behavior. We have already discussed the case of a burst extending over part of the barrier region and part of the downstream unstable region. An alternative case of interest is that of concomitant overdense burst and subdense burst. In the barrier region the overdense burst penetrates the barrier moving downstream, its electrostatic dipole decaying due to the barrier stabilization. Conversely, the subdense burst penetrates the barrier moving upstream and is characterized by an opposite dipole compared to the superdense burst. With appropriate phasing of the collision between the overdense burst and the subdense burst, one can then amplify the dipole thus providing another possible mechanism for the growth of these bursts.

\section{Transport barrier response to turbulent burst pene- tration in numerical simulations}

We analyze here those aspects of the interplay between the turbulent bursts and the barriers that are consistent with the stochastic processes proposed in the previous Section. The overall description of this transport barrier appears to fit with version $(T A)$, "take away", of Section 3. The first stochastic field corresponds to the barrier width. Provided one can properly evaluate the barrier width, and that the latter effectively corresponds to the region governed by a damping of the incoming structures, one can consider that it is possible to determine the time evolution and the distribution function of the barrier width.

In order to properly compare the actual barriers to the stochastic models, one must identify the the following characteristic quantities as stochastic processes:

- the barrier width and consequently the PDF of the radial extent $\Delta_{B}$ where turbulence is stabilized; 
- the stopping distance $\delta_{t b}$ of the turbulent bursts within the barrier and the PDF that governs this property;

- the relative time scale of fluctuation of $\Delta_{B}(t), \delta_{t b}(t)$ that determines in fact the degree of correlation between the incoming burst and the barrier width.

In Section 2 we have shown that the barrier width is readily observed to exhibit a randomness that we evaluate by the PDF of the quantity $R_{B}$. However, we also indicate that the actual region where turbulence is stabilized is not identical to the region where the turbulence transport actually decays, as estimated with the parameter $R_{B}$. In the following we shall assume however that these regions are comparable so that the PDF of the barrier width is appropriate to determine that of $\Delta_{B}$ where the turbulent bursts are stabilized. We thus rather concentrate on the case of the soft barrier where such a relation can be regarded as appropriate.

The second stochastic field that enters the analytical model is the position where the turbulent bursts are stopped. This location depends on the analysis of the trajectories of individual turbulent bursts and requires that one follows such trajectories based on structure recognition analysis that is not performed in these simulations. However, following the analysis given above, it appears to be reasonable to correlate the behavior of the stopping distance of the turbulent bursts $\delta_{t b}$ to the velocity of the incoming turbulent bursts $v_{t b}$. Previous structure recognition analysis have allowed us to determine the NDF of the turbulent bursts velocity for a standard turbulent simulation, here characterized by the ratio of the turbulent burst velocity and the sound speed (taken constant in these simulations), namely the Mach number $M$, see figure 11. The distribution of the Mach number of the density bursts is peaked in the positive Mach number region hence for turbulent bursts propagating from the source toward the distributed sink. It is skewed away from the gaussian fit at high velocities but the data do not allow one to discriminate an exponential tail with characteristic Mach number e-folding length of the order of 0.01 from a power law decay close to $M^{-1}$ followed by an exponential drop. Such a Mach number statistics of the turbulent bursts is

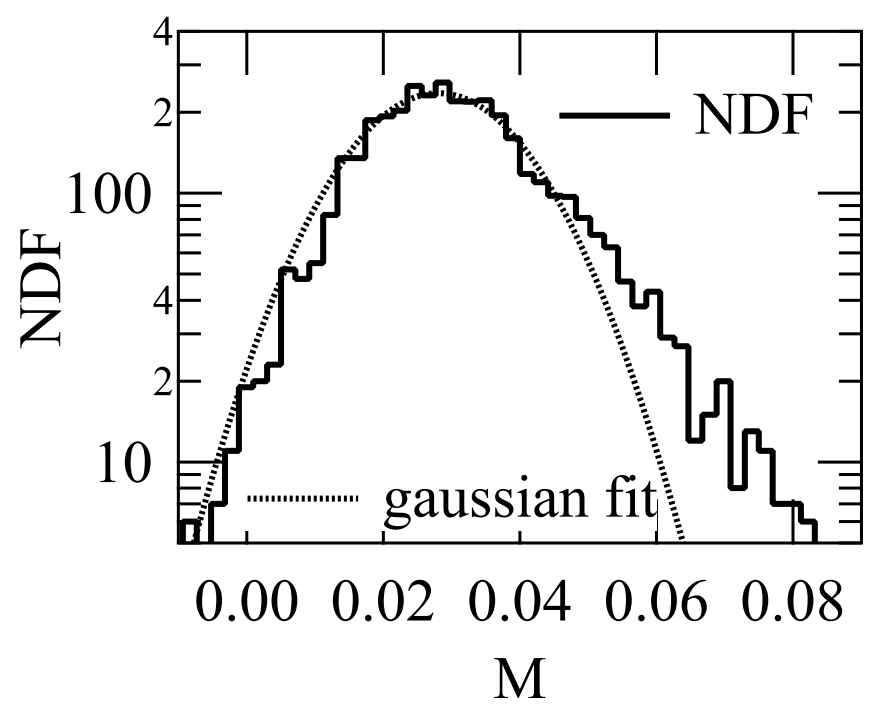

Figure 11: NDF of the turbulent burst velocity for a standard turbulent simulation. The dashed line represents a gaussian fit.

reminiscent of that of the particle flux. This is to be expected since the largest flux events 
are governed by the turbulent bursts themselves. Indeed, the heavy tail of the PDF of the particle flux is such that the events more than two standard deviations above the mean value drive $28 \%$ of the total particle flux. This correlation between the turbulent burst velocity and the radial particle flux is used here to estimate the statistical properties of the turbulent burst velocities on the basis of the PDF of the particle flux. We thus only consider the latter probability distribution function since the PDF of the turbulent burst velocity is not available in the present simulations (a case that is also met in many experiments). However, in order to retain the signature of the coherent turbulence bursts rather than the fluctuations of the particle flux, we consider the maximum of the particle flux, $\max (\Gamma)$, taken over the poloidal domain of the simulation and at given radial location. Although very imperfect, this data will be more closely related to the turbulent bursts since one can observe in these simulations that, at all times, there is typically of order of 1 turbulent burst at a given radial position and for all the poloidal positions in the simulation region. The time trace of

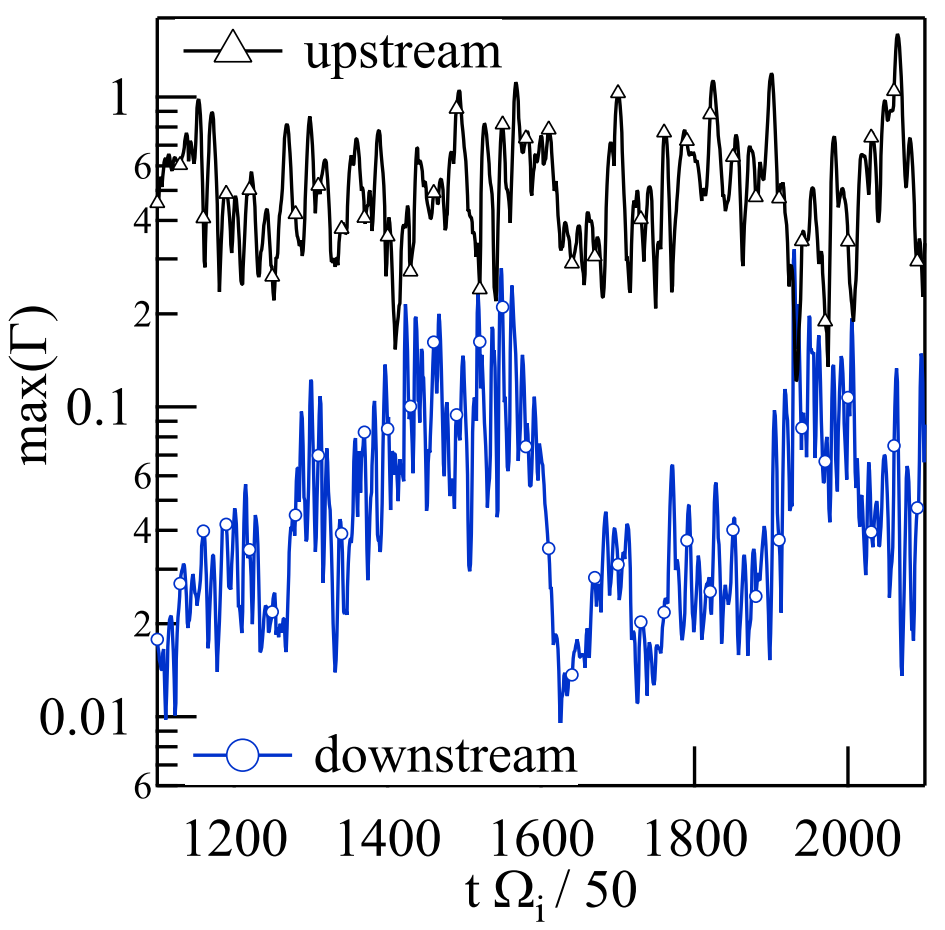

Figure 12: Time trace of the particle flux measured upstream and downstream from the barrier.

$\max (\Gamma)$, upstream and downstream from the barrier is plotted on figure 12 . Two important aspects are readily noticeable on this plot, first there is a sharp decrease of the particle flux across the barrier, second that no clear correlation can be observed between the two sides of the barrier. The first point is specific of the present model and is governed by the parallel loss of particles to the wall. The barrier, by reducing the turbulent transport, favors the parallel particle loss. While flux conservation is a reasonable assumption in the core plasma barriers, the SOL barrier is thus characterized by a saturation mechanism due to the parallel transport and leading to a decrease of the flux across the barrier. The apparent lack of correlation between the two sides of the barrier exemplified by the time trace could be due to the response time of the barrier. Indeed, given the characteristic turbulent burst velocities, there is a typical time required for a turbulent burst to reach the downstream point starting from the upstream position. Such a time lag can be noticed on figure 5 for which the Mach number of a particular event was estimated to be of the order of $2.5 \%$, hence 
in the most probable region of the radial Mach number PDF, figure 11. With this value, one can estimate the time shift between the upstream and downstream to be of the order of $40\left(50 / \Omega_{i}\right)$. Given the distribution of the turbulent burst velocities, one should expect some randomness in this shift. In the time traces, the lack of correlation is readily noticed since they are times of very low downstream flux with standard upstream values. These results suggest that the barrier is weakly correlated with the tubulents bursts. The NDF of

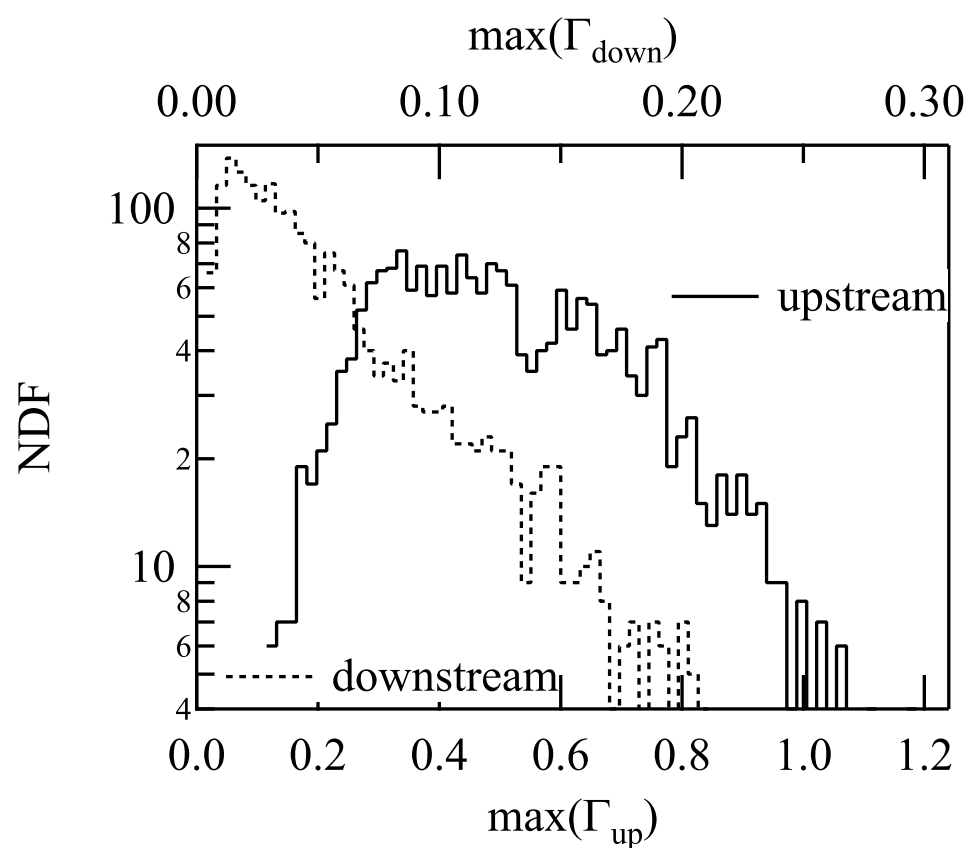

Figure 13: NDF of the turbulent particle flux shown figure 12. Note the factor 3 difference between the upper and lower scales.

$\max (\Gamma)$, plotted on figure 13, underlines the drop of particle flux within the barrier. The mean value of $\max (\Gamma)$ is found to decrease from $5.310^{-1}$ to $6.610^{-2}$, close to a factor 10 and similarly for the standard deviation that decreases from $2.110^{-1}$ upstream to $5.310^{-2}$ downstream. A major property of such SOL transport barriers is therefore to modify the balance between the parallel and transverse transport leading to enhance parallel losses and consequently some form of "isolation" of the downstream region. This is why it has been proposed to implement a biasing system to generate such transport barriers and screen the main chamber wall from ELM induced energy flux [27].

Let us now consider the NDF of $\max (\Gamma)$ that we use as a measure of the barrier effect on the turbulent burst. Regarding the NDF of the upstream value of $\max (\Gamma)$, one finds that there is no clear trend in the heavy tail decay rate. Exponential or power law decays are compatible with the data as well as combinations of the two behaviors. However, regarding the NDF of the downstream quantity one finds that the decay can be considered as closer to an exponential decay extending from the smallest value to the largest. This PDF thus appears to correspond to a Poisson law with a threshold value. The e-folding of the NDF, corresponds to a particle flux of $6.210^{-2}$ (in the units used in the code, arbitrary units for the density and velocity normalized by the sound speed). This value can readily be compared to the time- averaged value of $\max (\Gamma)$ at this location, $6.610^{-2}$, as well as to the root mean square of the fluctuations of $\max (\Gamma), 5.310^{-2}$. The ratio of the mean value to that of the e-folding length is compatible with the exponential shape of the NDF with a threshold value of 0.1 in $\max (\Gamma)$. For an exponential PDF, one also expects the root mean square to be 
equal to the e-folding length. Values that are obtained in the analysis are consistent with these constraints and thus support the analysis of the downstream PDF as being exponential.

The shearing barrier studied numerically thus appears to exhibit several of the key features introduced in the stochastic models presented in Section 3. The latter then provide a guide line to determine the relevant parameters that should be measured as well as a means to interpret the results. Considering the case of the barrier obtained by biasing the plasma boundary, we have found that the turbulent bursts impinging into the barrier exhibit a PDF that is similar for their amplitude and their velocity. Further simulations would be required to investigate precisely this relationship. For the stochastic model presented here, the key point is that the turbulent burst velocity that we assume to control the stopping distance in the barrier appears to be governed by a stochastic process with a measurable PDF. A second point that has been analyzed is that the barrier width and the efficiency of the barrier also appear to fluctuate in time. While the former is consistent with the stochastic behavior of the barrier width introduced in Section 3, the latter can be combined to the fluctuations of the stopping distance of the turbulent bursts and can be viewed as leading to an effective PDF of the stopping distance combining the fluctuations of the turbulent bursts to that of the barrier stopping capability. The present analysis of the shearing barrier does not appear to exhibit behaviors that are reminiscent of model (CG), cross and grow presented in Section 3.2, although dedicated simulations would be required to fully assess this point. In the framework of model (TA), take away model in Section 3.1, one must identify a further parameter to bridge the analytical model and the simulations, namely the parameter labeled $\eta$ that measures the coupling between the incoming turbulent bursts and the fluctuations of the barrier width, in particular with respect to their characteristic time scales. Comparing figures 6 and 12, the time traces of the barrier width $\Delta_{B}(t)$ and the turbulent burst stopping distance, assuming that it behaves like $\max (\Gamma)$ at the upstream position, one finds comparable time scales, figure 14. In both cases, one finds a broad spectrum with very sim-

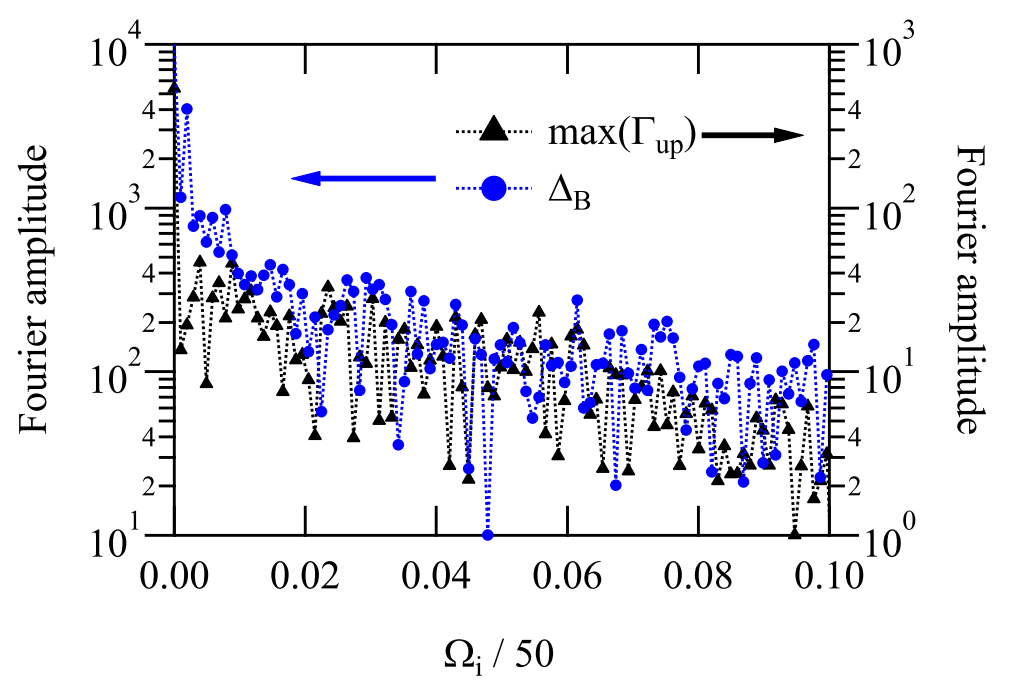

Figure 14: Amplitude of the Fourier modes for the Barrier width, left hand scale and of $\max \left(\Gamma_{u p}\right)$ that stands for the turbulent burst stopping distance, right hand scale.

ilar shapes and no outstanding frequency. Such a spectrum is characteristic of signals with bursts and do not allow one to investigate possible quasi-coherent behaviors. However, they provide a clear indication that the short time scales (high frequency) of the two stochastic processes are comparable. This suggests that our model should be applicable with a value 
of parameter $\eta$ close to 0 . If this is the case, the results of [47] tell us that we should have an exponential decay for the large energy release, that is the barrier is a leaky barrier. This appears to agree with the amplitude of the turbulent bursts transmitted by the barrier insofar that one considers that $\max \left(\Gamma_{\text {down }}\right)$ provides an appropriate measure of the turbulent burst magnitude transmitted by the barrier, figure 13. Furthermore, given the parallel loss that leads to a saturation of the density build-up in the barrier, coining such a barrier as a leaky barrier from the stochastic point of view agrees with the physics at work within the barrier. The stochastic model "take away" thus appears to provide the appropriate approach to the interplay between the turbulent bursts and transport barriers triggered by external edge biasing. However, the volumetric loss within the barrier is such that there is little to take away. When analyzing the low frequency part of the spectra in figure 14 one finds that the barrier evolution exhibits slower time scales than the turbulent bursts. This result could indicate a direction for more complex stochastic models with a twofold characterization of the barrier. Part of the barrier would exhibit a slow evolution weakly correlated to the turbulent bursts and accounting for a nearly constant contribution to the barrier width. The other part would be characterized by a rapid adjustment of the barrier width to the burst activity, the relative width of the slow and fast contributions to the overall barrier width being a further parameter of the system. It is noteworthy that the latter system would screen out all incoming bursts below a given magnitude. This would very likely govern a downstream PDF with a sharp rise, like a threshold effect, followed by an exponential decay, as readily observed in figure 13.

\section{Discussion and conclusion}

Transport barriers in magnetic fusion plasmas are key confinement structures for all magnetic configurations. In flux driven regimes, turbulent transport appears to be strongly linked to coherent turbulent structures that exhibit an intermittent behavior. These structures are observed in simulations, fluid and gyrokinetic, as well as in experiments. In this paper we point out that the barrier and the turbulent bursts form a self-consistent system fluctuating in time and space. We propose a stochastic framework for analyzing and interpreting the dynamics of such a system. As an input to the various stochastic models we are led to identify and "measure" key parameters that govern the physics in the stochastic framework. Provided these can be determined, theoretically or in simulations, we can then estimate with the stochastic models the performance of the barrier, in particular the probability distribution function of outgoing turbulent bursts, either due to relaxation events or driven by turbulent bursts that burn through the barrier.

The two main physical quantities relevant in the stochastic models analyzed in this paper are the radial extent of the barrier domain and the velocity of the incoming turbulent bursts. The latter is considered to be important as a quantity that determines the stopping distance of the turbulent burst inside the barrier. On the basis of the statistical properties of these two variables, the model provides the form of the tail of the PDF of the amplitude of the escaping turbulent bursts. In particular it gives conditions to obtain tight barriers, hence barriers with heavy tails with power law scaling. The occurrence of large magnitude relaxation events will then occur with a significant probability. Conversely, the leaky barriers, with frequent relaxation events or outgoing turbulent bursts, will lead to exponential tails.

Simulations of transport barriers are presented and used as a test-bed for the stochastic approach. These simulations are based on a set of two coupled fluid balance equations for plasma turbulence in the SOL region. Numerical simulations for this system are performed with the TOKAM-2D code. The transported quantity is the plasma density. A specific feature of the analysis of SOL transport is the existence of parallel particle losses to the wall everywhere in the simulation domain and therefore also in the pedestal. Such a particle loss plays the role of a volumetric loss term and governs the saturation mechanism for the 
pedestal. Simulations of two types of barriers have been achieved. In the so-called hard barriers the $g$-term that drives the interchange turbulence is artificially switched-off in a given radial region. Plasma biasing is used to drive a soft barrier, namely a barrier where the plasma response plays a role in establishing the barrier properties. The stochastic model in Section 3 then provides a guideline for analyzing and interpreting the simulation data.

In the first case, the "hard barrier", the barrier is fully determined since the $g=0$ region with no drive and therefore, governed by turbulence stabilization, is fixed by the simulation parameters. This model allows one to test a criterion providing a way of localizing the barrier. The indicator labeled $R_{B}$ is the ratio $\Gamma_{\text {turb }} / \Gamma_{\text {tot }}$. As we have shown, this criterion corresponds to a standard experimental approach defining a barrier with respect to a threshold value for relevant (for example density) gradient scales. The surprising result is that the barrier domain identified using the $R_{B}$ indicator fluctuates in time whereas the region where the turbulence drive term is set to zero is fixed in time. The mechanism responsible for such an effect is the spreading of turbulence in stable regions. As a consequence one observes a residual turbulent transport up to the center of the barrier domain and an effective shape of the barrier with a characteristic bell shape for the transport reduction.

In the second case, that of the "soft barrier", the induced barrier region is not fully determined due to the significant plasma modification of the electrostatic potential from the value induced by the external biasing. The indicator $R_{B}$ is used to investigate the barrier width. The simulations indicate that the barrier fluctuates in width and in efficiency. In order to analyze the properties of the turbulent bursts, whether upstream or downstream, we have built as characteristic signal, the maximum of the radial particle flux, the maximum being taken over all poloidal values. The argument in favor of such a marker is that the particle flux strongly depends on the bursts of turbulence transport. The maximum over the poloidal domain of the simulation allows one to reinforce the weight of the turbulent bursts in the marker. Such a treatment of the data remains reasonable insofar that typically only one turbulent burst exists in the poloidal region of the simulation at a given time and given radial location. The $\mathrm{PDF}$ of the upstream value of the marker $\max (\Gamma)$ is characterized by heavy tails. The data does not allow one to discriminate between power law tails or exponential tails, or a combination of the two. However, we use this marker to analyze the correlation between the barrier fluctuations and the turbulent burst fluctuations. For the present simulations, one finds that both variables evolve with the same time scales which corresponds to the framework of model (TA) "take away" with $\eta$ close to 0 . The downstream value of the marker is used to analyze the magnitude of the escaping turbulent bursts. In agreement with the expectation of a leaky barrier, the PDF of the escaping turbulent bursts is found to be clearly exponential.

The stochastic models presented in this paper provide an efficient framework to analyze transport barriers. They allow one to determine key parameters that help to investigate the physics of the transport barriers. Furthermore, their predictions regarding the heavy tails of the probability distribution functions of the magnitude of the escaping turbulent bursts can be compared to actual data, experimental and from simulations. It is to be underlined that the method we propose is based on the measurement of existing turbulent burst propagation and the interpretation of the measured PDFs. As a proof of principle, we have used available simulations and their diagnostics. We have then developed signals that allow us to estimate the relevant properties of the stochastic variables. The first comparisons are interesting and show that the interplay between the bursts of turbulent transport and the transport barriers can be framed in a stochastic approach where the barrier is one of the stochastic variables. This point of view is in contrast with the usual description as slowly evolving structures. We believe that it offers an interesting means to revisit our understanding of the physics of the transport barriers. Two important issues could be addressed with the PDF analysis in the framework of the stochastic models. First, one would obtain a measure of the quality of the barrier, tight or leaky, and therefore a means to differentiate the barriers. Second, this approach allows one to address the barriers as time dependent structures with micro-scale 
evolution governed by the turbulent bursts and relaxation events at the meso-scale times. On the basis of the PDF analysis proposed in this paper, one can also expect macro-time scale evolution. These results suggest that the most likely situations is that the barrier behaves like a leaky barrier for the very large relaxation events. As a consequence, barrier termination governed by a very strong relaxation could be ruled out, while barrier erosion and soft termination cannot be discarded. However, such key issues for burning plasma operation cannot be addressed with present simulations that do not exhibit self-consistent barriers. This underlines the need for an ongoing effort, both theoretical and experimental, to improve our understanding this physics, both on the turbulence time scale and on the long times of pseudo steady-states.

Acknowledgments. This work was supported by the European Community under the contract of Association between EURATOM, CEA, and the French Research Federation for fusion study. The views and opinions expressed herein do not necessarily reflect those of the European Commission.

\section{A Some details on the computation of the PDF of out- going energy in (TA) Model "take away"}

We recall here some results on the computation of $\mathcal{P}_{N}\left(N_{i n}\right)$ carried out in [47], using the notations proper to our situation. Let us introduce the cumulative probabilities:

$$
\begin{gathered}
F(x)=\mathbb{P}\left\{\delta_{t b}<x\right\}=\int_{0}^{x} d \delta_{t b} \mathcal{P}_{\delta}\left(\delta_{t b}\right) \\
G(y)=\mathbb{P}\left\{\Delta_{B}<y\right\}=\int_{0}^{y} d \Delta_{B} \mathcal{P}_{\Delta}\left(\Delta_{B}\right)
\end{gathered}
$$

$F$ and $G$ satisfy the normalization conditions $F(0)=G(0)=0, F(L)=G(L)=1$.

Each turbulent burst crossing the barrier can either be trapped (event $T$ ) or escape (event $E$ ). Events $T, E$ can take place either in the resting or asynchronous way $r$ (the width of the barrier $\Delta_{B}$ keeps the value it had at the passage of the preceding turbulent burst), or in the modified or synchronous way $m$ (the width of the barrier is chosen with $\operatorname{PDF} \mathcal{P}_{\Delta}\left(\Delta_{B}\right)$ ).

Let us consider the event $N_{i n}=0$, i.e., the first turbulent burst passing in the barrier after an energy release escapes. In this case, as stated in the definition of the model in section 3.1 , the width $\Delta_{B}$ of the barrier readjusts systematically. The probability of that $N_{\text {in }}=0$ is, since the random variables $\delta_{t b}, \Delta_{B}$ are taken to be independent:

$$
\mathcal{P}_{N}(0)=\mathbb{P}\left[E_{m}\right]=\int_{0}^{L} d G(y) \int_{y}^{L} d F(y)=\int_{0}^{L} d G(y)(1-F(y))=1-\mathbb{P}\left[T_{m}\right]
$$

Each phase of energy accumulation in the barrier, ended by an energy release, corresponds to a sequence of events $T_{r}, T_{m}$, ended by an escape $E_{r}$ or $E_{m}$, weighed by the appropriate probability. We recall that subsequent trappings and escapes $\left(N_{i n} \geq 1\right)$ take place in the resting way $r$ with probability $\eta$, or in the modified way $m$ with probability $1-\eta$.

Defining, for $n=0,1,2, \ldots$,

$$
A(n)=\int_{0}^{1} d G(y) F(y)^{n}
$$


and

$$
B(n)=\int_{0}^{1} d G(y) F(y)^{n}(1-F(y))=A(n)-A(n+1)
$$

we have

$$
\begin{aligned}
\mathcal{P}_{N}(1) & =\mathbb{P}\left[T_{m} E_{r}\right]+\mathbb{P}\left[T_{m} E_{m}\right] \\
& =\int_{0}^{L} d G(y) F(y) \eta(1-F(y)) \\
& +\int_{0}^{L} d G(y) F(y)(1-\eta) \int_{0}^{L} d G(z)(1-F(z))= \\
& =\eta B(1)+(1-\eta) A(1) B(0)
\end{aligned}
$$

Similarly,

$$
\begin{aligned}
\mathcal{P}_{N}(2) & =\mathbb{P}\left[T_{m} T_{r} E_{r}\right]+\mathbb{P}\left[T_{m} T_{m} E_{r}\right]+\mathbb{P}\left[T_{m} T_{r} E_{m}\right]+\mathbb{P}\left[T_{m} T_{m} E_{m}\right] \\
& =\eta^{2} B(2)+\eta(1-\eta) A(1) B(1)+\eta(1-\eta) A(2) B(0)+(1-\eta)^{2} A(1)^{2} B(0)
\end{aligned}
$$

One can show that the generating function of $\mathcal{P}_{N}\left(N_{i n}\right)$, defined by

$$
\hat{\mathcal{P}}(s)=\sum_{N_{i n}=0}^{\infty} s^{N_{i n}} \mathcal{P}_{N}\left(N_{i n}\right)
$$

can be written

$$
\hat{\mathcal{P}}(s)=B(0)+\rho s+\frac{s}{1-\hat{p}(s) \hat{q}(s)}[\hat{r}(s)+\rho \hat{p}(s) \hat{q}(s)+\rho A(1) \hat{q}(s)+A(1) \hat{q}(s) \hat{r}(s)]
$$

where $\hat{p}(s), \hat{q}(s), \hat{r}(s)$ are the generating functions of the auxiliary functions $p(l), q(l), r(l)$ :

$$
\begin{gathered}
p(l)=\eta^{l} A(l+1), \quad \text { for } l \geq 1, \quad p(0)=0 \\
q(l)=(1-\eta)^{l} A(1)^{l-1}, \quad \text { for } l \geq 1 \quad q(0)=0 \\
r(l)=\eta^{l}[\eta B(l+1)+(1-\eta) A(l+1) B(0)], \quad \text { for } l \geq 1, \quad r(0)=0
\end{gathered}
$$

and $\rho$ is defined by

$$
\rho=\eta B(1)+(1-\eta) A(1) B(0)
$$

The generating property of the function $\hat{\mathcal{P}}(s)$ is such that

$$
\mathcal{P}\left(N_{i n}\right)=\left.\frac{1}{N_{i n} !} \frac{d^{N_{i n}} \hat{\mathcal{P}}(s)}{d s^{N_{i n}}}\right|_{s=0}
$$

which allows to recover $\mathcal{P}\left(N_{i n}\right)$ from the knowledge of $\hat{\mathcal{P}}(s)$.

In the marginal cases $\eta=0$ and $\eta=1$, the probability $\mathcal{P}\left(N_{i n}\right)$ can be readily calculated. For $\eta=0$ we have

$$
\mathcal{P}_{\eta=0}\left(N_{i n}\right)=A(1)^{N_{i n}} B(0)=\left[\int_{0}^{1} d G(y) F(y)\right]^{N_{i n}} \int_{0}^{1} d G(y)(1-F(y)) .
$$

Therefore, in this case, for any choice of the distributions $F$ and $G$ having supports with non empty intersections, the probability $\mathcal{P}\left(N_{\text {in }}\right)$ decays exponentially.

For $\eta=1$ :

$$
\mathcal{P}_{\eta=1}\left(N_{i n}\right)=B\left(N_{i n}\right)=\int_{0}^{1} d G(y) F(y)^{N_{i n}}(1-F(y))
$$


One can give upper and lower bounds for $\mathcal{P}\left(N_{i n}\right)$, for any value of the parameter $\eta$, namely

$$
\begin{aligned}
\mathcal{P}_{\eta}\left(N_{i n}\right) & \leq \eta^{N_{i n}} B\left(N_{i n}\right)+(1-\eta) A(1) B(0)[\eta+(1-\eta) A(1)]^{N_{i n}-1} \\
& +\eta A(1)\left\{[\eta+(1-\eta) A(1)]^{N_{i n}-1}-\eta^{N_{i n}-1}\right\}
\end{aligned}
$$

and

$$
\begin{aligned}
\mathcal{P}_{\eta}\left(N_{i n}\right) & \geq \eta^{N_{i n}} B\left(N_{i n}\right)+(1-\eta) A(1)^{N_{i n}} B(0) \\
& =\eta^{N_{i n}} \mathcal{P}_{\eta=1}\left(N_{i n}\right)+(1-\eta) \mathcal{P}_{\eta=0}\left(N_{i n}\right)
\end{aligned}
$$

We thus see that, for any $0 \leq \eta<1$, the decay of distribution $\mathcal{P}\left(N_{i n}\right)$ is bounded by exponentials. On the contrary, in case $\eta=1$, we are able to construct a large family of distributions $F$ and $G$ giving rise to polynomial statistics of the event $N_{i n}$. For the intermediate case $0<\eta<1$ it is shown that when $\delta_{t b}$ and $\Delta_{B}$ have uniform distributions, the statistics of $N_{i n}$ is polynomial during large times, scaling as $(1-\eta)^{-1}$, and, only for asymptotic limits it becomes exponential.

\section{B Determining the heavy tail behavior in $(C G)$ Model "cross and grow"}

The mechanism generating the growth of the final amplitude at the end of the transport barrier is assumed to depend on the velocity that is achieved when reaching the amplification region. This velocity depends on the interplay between $\Delta_{c}$, the extent of the stabilizing region, and $\delta_{t b}$, the stopping distance of the turbulent burst. Since the velocity is assumed to be constant in the second region where amplification occurs, the most important parameter in the asymptotic regime is the velocity achieved at the end of the stabilization region, the largest effects being obtained when the stopping distance $\delta_{t b}$ is close but larger than $\Delta_{c}$. The heavy tale of the distribution of the ratio of the amplitude of the turbulent burst leaving the barrier $A_{\text {out }}$ versus the amplitude of the turbulent burst entering the barrier $A_{\text {in }}$ will then be governed by the values such that $1-\Delta_{c} / \delta_{t b} \rightarrow 0^{+}$. Since we assume that the turbulent burst velocity goes to zero, we can thus simplify the calculations of the time spent in each region by using the linear variation of the turbulent burst velocity in the vicinity of zero. We thus assume:

- a linear approximation of the turbulent burst velocity profile $v(x)$ not only near the end of the barrier, but for the entire decreasing part of $v(x)$ (see figure 15);

- focusing on the dependence on the lag $\delta=\left(\delta_{t b}-\Delta_{c}\right)$ between the turbulent burst stopping distance and the width of the stabilizing region.

We take a linear approximation for $v(x)$, calling $d$ the distance in the barrier where the turbulent burst velocity stays constant before starting to decrease linearly, $v(x)=v_{t b}$. Alternatively $d$ can stand for the distance where the turbulent burst velocity $v(x)$ is large enough that we can neglect its variation with respect to the divergences of the asymptotic limit. If $\Delta_{c} \leq \delta_{t b}$ we then obtain:

$$
t_{c}=\frac{\delta_{t b}}{v_{t b}} \ln \left(\frac{\delta_{t b}}{\delta_{t b}-\Delta_{c}}\right)
$$

where $t_{c}$ is the time taken to cross region one of extent $\Delta_{c}$ with a velocity linearly decreasing to zero. This calculation is only valid for $\delta_{t b}-\Delta_{c}>0$ otherwise $t_{c} \rightarrow+\infty$ since the turbulent burst is trapped in the barrier. This calculation of the time lag within the first region is 


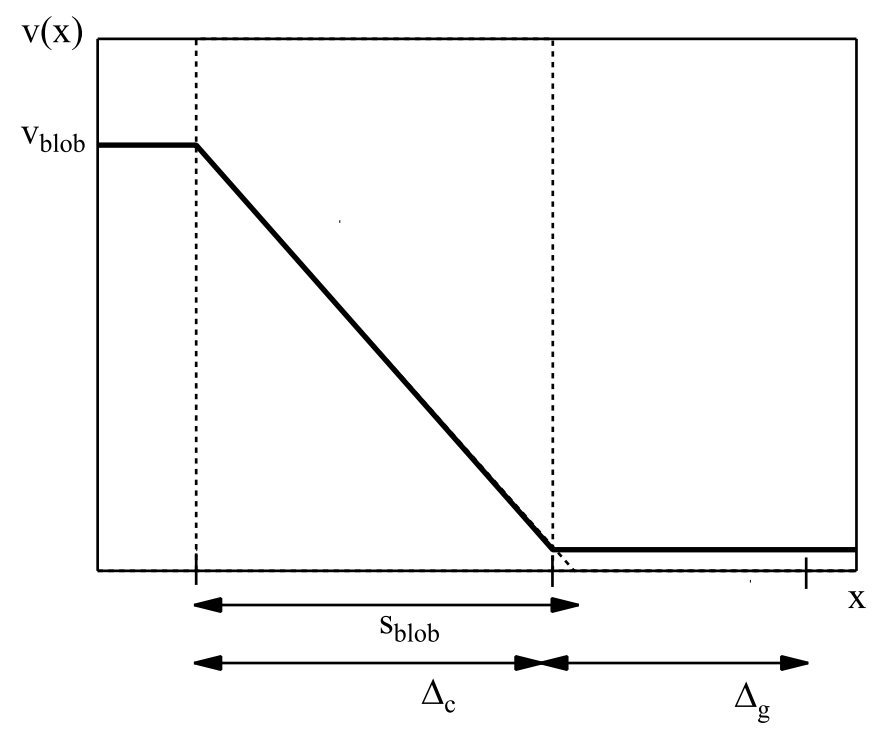

Figure 15: Linear approximation of the velocity profile.

based on the linear variation of $v(x)$ that also allows one to compute the velocity of the turbulent burst $v_{g}$ in the second region of the barrier, where the turbulent burst amplitude grows and where one assumes that the turbulent burst velocity is constant.

$$
v_{\text {out }}=v_{t b} \frac{\delta_{t b}-\Delta_{c}}{\delta_{t b}}
$$

Finally, we can compute the lag time in the second region of extent $\Delta_{g}$ where the turbulent burst amplitude grows:

$$
t_{g}=\frac{\Delta_{g}}{v_{\text {out }}}=\frac{\Delta_{g}}{v_{t b}} \cdot \frac{\delta_{t b}}{\delta_{t b}-\Delta_{c}}
$$

Defining, for the case of turbulent bursts passing the barrier, the positive random variable $z=\frac{\delta_{t b}-\Delta_{c}}{\delta_{t b}}$, which represents a normalized distance to threshold, we get:

$$
A_{\text {out }}(z)=A_{\text {in }} f_{c}\left(\frac{\delta_{t b}}{v_{t b}} \ln \left(\frac{1}{z}\right)\right) f_{g}\left(\frac{\Delta_{g}}{v_{t b}} \cdot \frac{1}{z}\right)
$$

We now explicitly discuss the cases:

b1) $f_{c}$ exponentially decreasing, $f_{g}$ exponentially increasing

We have:

$$
f_{c}(t)=e^{-\gamma_{c} t}, \quad f_{g}(t)=e^{\gamma_{g} t}
$$

where $\gamma_{c}, \gamma_{g}$ are positive constants. We define

$$
\begin{gathered}
w=\frac{A_{\text {out }}}{A_{\text {in }}} \\
\beta_{c}=\frac{\gamma_{c} \delta_{t b}}{v_{t b}}>0, \quad \beta_{g}=\frac{\gamma_{g} \Delta_{g}}{v_{t b}}>0
\end{gathered}
$$


$w$ is the interesting quantity for our problem since it represents the amplitude gain due to the subsequent passage in the transport barrier and in the turbulent region, i.e. the ratio between the outgoing ant ingoing amplitudes of the turbulent burst. We get for:

$$
w=\exp \left(\beta_{c} \ln z+\frac{\beta_{g}}{z}\right)
$$

We would like to evaluate the $\operatorname{PDF} \mathcal{P}_{W}(w)$ of $w$, or at least its decay as $w \rightarrow+\infty$. $\mathcal{P}_{W}(w)$ satisfies:

$$
\mathcal{P}_{W}(w)=\mathcal{P}_{Z}(z)\left|\frac{d z}{d w}\right|=\mathcal{P}_{Z}(z(w)) \frac{z(w)^{2}}{\beta_{g} w}\left|1-\frac{\beta_{c}}{\beta_{g}} z(w)\right|^{-1}
$$

The function $w(z)$ has a minimum for $z=\beta_{g} / \beta_{c}$ and goes to infinity as $z$ goes to zero. This means that, for sufficiently small $z$ (i.e., for the turbulent burst stopping distance $\delta_{t b}$ sufficiently close to the barrier width $\Delta_{c}$ ) we will have that:

(i) $w(z)$ is monotonically decreasing and thus invertible and

(ii) $\beta_{c} \ln z \ll \beta_{g} / z$, so that $w(z) \simeq \exp \left(\beta_{g} / z\right)$

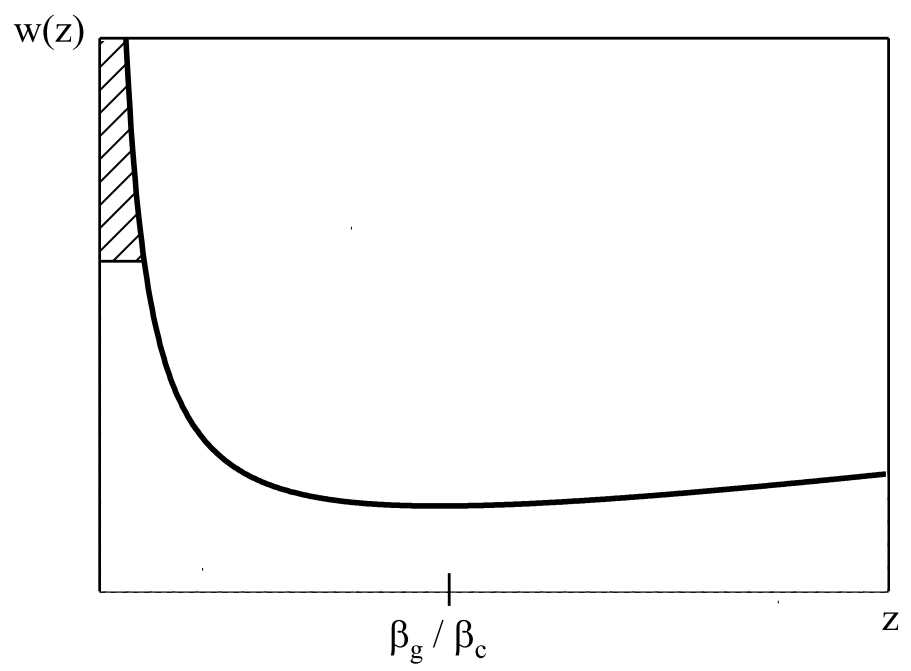

Figure 16: The behavior of the function $w(z)$ defined by (6).

So, for sufficiently large $w$, we have $z(w) \simeq \beta_{g} / \ln w$ and

$$
\mathcal{P}_{W}(w) \approx \mathcal{P}_{Z}\left(z=\beta_{g} / \ln w\right) \frac{\beta_{g}}{w(\ln w)^{2}}
$$

For example, in the case where $\mathcal{P}_{Z}$ is uniform, $\mathcal{P}_{W}(w)$ decays as

$$
\mathcal{P}_{W}(w) \propto \frac{1}{w \ln ^{2} w}
$$

so that all the moments of $\mathcal{P}_{W}(w)$ diverge.

b2) $f_{c}$ exponentially decreasing, $f_{g}$ increasing as a power law 
We have:

$$
f_{c}(t)=e^{-\gamma_{c} t}, \quad f_{\text {out }}(t)=\left(1+\gamma_{g} t\right)^{\lambda_{g}}
$$

where $\gamma_{c}, \gamma_{g}, \lambda_{g}$ are positive constants. As in Section b1, this leads to study the PDF of the amplitude gain $w$ :

$$
w=\frac{A_{\text {out }}}{A_{\text {in }}}=\exp \left[\beta_{c} \ln z+\lambda_{g} \ln \left(1+\frac{\beta_{g}}{z}\right)\right]
$$

where $\beta_{c}$ and $\beta_{g}$ are defined above. We see that the non trivial case where $w$ can take arbitrarily large values:

$$
\lim _{z \rightarrow 0^{+}} w=+\infty
$$

corresponds to choosing $\lambda_{g}>\beta_{c}$ since $w \approx z^{\beta_{c}-\lambda_{g}}$ for $z \rightarrow 0^{+}$. The $\operatorname{PDF} \mathcal{P}_{W}(w)$ of $w$ satisfies:

$$
\mathcal{P}_{W}(w)=\mathcal{P}_{Z}(z)\left|\frac{d z}{d w}\right|=\mathcal{P}_{Z}(z(w)) \frac{z(w)}{w} \frac{1}{\left|\beta_{c}-\frac{\lambda_{g} \beta_{g}}{z(w)+\beta_{g}}\right|}
$$

For $\lambda_{g}>\beta_{c}$ and $z$ sufficiently small we will then have:

(i) $w(z)$ is monotonically decreasing and thus invertible and

(ii) $w(z) \simeq z^{\beta_{c}-\lambda_{g}}$

So, for sufficiently large $w$, we have $z(w) \simeq w^{\frac{1}{\beta_{c}-\lambda_{g}}}$ and

$$
\mathcal{P}_{W}(w) \approx \mathcal{P}_{Z}\left(z=w^{\frac{1}{\beta_{c}-\lambda_{g}}}\right) \frac{w^{-\left(1+\frac{1}{\lambda_{g}-\beta_{c}}\right)}}{\lambda_{g}-\beta_{c}}
$$

For example, in the case where $\mathcal{P}_{Z}$ is uniform, $\mathcal{P}_{W}(w)$ decays as

$$
\left.\mathcal{P}_{W}(w) \propto w^{-\left(1+\frac{1}{\lambda_{g}-\beta_{c}}\right.}\right)
$$

so that all the moments of $\mathcal{P}_{W}(w)$ of order $n \geq n_{0}$ diverge, where $n_{0}$ is the smallest integer such that $\lambda_{g}-\beta_{c}>1 / n_{0}$.

\section{References}

[1] M. Shimada, D.J. Campbell, V. Mukhovatov, M. Fujiwara, N. Kirneva et al., Progress in the ITER Physics Basis, Chapter 1: Overview and summary, Nucl. Fusion 47, S1 (2007).

[2] F. Wagner A quarter-century of H-mode studies, Plasma Phys. Control. Fusion 49 B1 (2007).

[3] E.J. Doyle, W.A. Houlberg, Y. Kamada, V. Mukhovatov, T.H. Osborne et al., Progress in the ITER Physics Basis, Chapter 2: Plasma confinement and transport, Nucl. Fusion 47, S18 (2007).

[4] P H Diamond, S-I Itoh, K Itoh and T S Hahm, Zonal flows in plasma - a review, Plasma Phys. Control. Fusion 47, R35 (2005).

[5] A. Fujisawa Experimental studies of mesoscale structure and its interactions with microscale waves in plasma turbulence, Plasma Phys. Control. Fusion 53124015 (2011).

[6] S.W. Yoon, J.-W. Ahn, Y.M. Jeon, T. Suzuki, S.H. Hahn et al., Characteristics of the first H-mode discharges in KSTAR, Nucl. Fusion 51113009 (2011). 
[7] J.W. Connor, T. Fukuda, X. Garbet, C. Gormezano, V. Mukhovatov, M.Wakatani, the ITB Database Groupa and the ITPA Topical Group on Transport and Internal Barrier Physics, A review of internal transport barrier physics for steady-state operation of tokamaks, Nucl. Fusion 44, R1 (2004).

[8] K.H. Burrell, Turbulence behaviour in the presence of transport barriers, Plasma Phys. Control. Fusion 48, A347 (2006).

[9] G.W. Pacher et al., Dependence of the $L$-H transition on separatrix-wall gaps on TdeV, J. Nucl. Mat. 266-296, 911 (1999).

[10] K. Kamiya et al., Observation of High Recycling Steady H-mode edge and compatibility with improved core confinement mode on JFT-2M, Nucl. Fusion 43, 1214 (2003).

[11] G. L. Jackson, J. Winter, T. S. Taylor, C. M. Greenfield, K. H. Burrell et al., Very high confinement discharges in DIIID after boronization, Phys. Fluids B 42181 (1992).

[12] T.H. Osborne, K.H. Burrell, T.N. Carlstrom, M.S. Chu, J.C. DeBoo et al., Confinement and stability of VH mode discharges in the DIII-D tokamak, 3523 (1995).

[13] ITER Physics Expert Groups on Confinement and Transport and Confinement Modelling and Database, ITER Physics Basis Editors ITER Physics Basis, Chapter 2: Plasma confinement and transport, Nucl. Fusion 39, 2175 (1999).

[14] P.W. Terry, Suppression of turbulence and transport by sheared flow, Rev. Mod. Phys. 72109 (2000).

[15] Y. Sarazin, V. Grandgirard, J. Abiteboul, S. Allfrey, X. Garbet et al., Predictions on heat transport and plasma rotation from global gyrokinetic simulations, Nucl. Fusion 51103023 (2011).

[16] Y. Sarazin and Ph. Ghendrih, Intermittent particle transport in two-dimensional edge turbulence, Physics of Plasmas 5, 4214 (1998).

[17] S.I. Krasheninnikov, On scrape off layer plasma transport, Physics Letters A 283, 368 (2001).

[18] D. A. D'Ippolito, J. R. Myra, and S. J. Zweben Convective transport by intermittent turbulent burst-filaments: Comparison of theory and experiment Phys. Plasmas 18 060501 (2011).

[19] Y. Sarazin et al., Large scale dynamics in flux driven gyrokinetic turbulence, Nuclear Fusion 50, 054004 (2010).

[20] S. Jolliet and Y. Idomura, Plasma size scaling of avalanche-like heat transport in tokamaks, Nucl. Fusion 52023026 (2012).

[21] T. S. Hahm and K. H. Burrell, Flow shear induced fluctuation suppression in finite aspect ratio shaped tokamak plasma, Phys. Plasmas 21648 (1995).

[22] R. E. Waltz, G. M. Staebler, W. Dorland, G. W. Hammett, M. Kotschenreuther, and J. A. Konings, A gyro-Landau-fluid transport model, Phys. Plasmas 42482 (1997).

[23] P. A. Politzer, Observation of Avalanche like Phenomena in a Magnetically Confined Plasma, Phys. Rev. Lett. 84, 1192 (2000).

[24] N. Fedorczak , J.P. Gunn, Ph. Ghendrih, G. Ciraolo, H. Bufferand, L. Isoardi, P. Tamain, P. Monier-Garbet, Experimental investigation on the poloidal extent of the turbulent radial flux in tokamak scrape-off layer, J. Nucl. Mater. (2010), doi:10.1016/j.jnucmat.2010.10.034

[25] Ph. Ghendrih, Y. Sarazin, G. Attuel et al., Statistical analysis of turbulent front propagation in the scrape-off-layer J. Nucl. Mater. 337-339 347 (2005).

[26] Ph. Ghendrih, P. Kaw, Y. Sarazin, P. Beyer, S. Benkadda, G. Falchetto, X. Garbet, V. Grandgirard, M. Ottaviani Scaling Intermittent Cross-Field Particle Flux to ITER, 20th IAEA Fusion Energy Conference 2004, Villamoura, Portugal, http://wwwnaweb.iaea.org/napc/physics/fec/fec2004/papers/th_1-3ra.pdf 
[27] Ph. Ghendrih et al., Theoretical analysis of the influence of external biasing on long range turbulent transport in the Scrape Off Layer, Nuclear Fusion 43, 1013-1022 (2003).

[28] J P Graves, J Horacek, R A Pitts and K I Hopcraft, Self-similar density turbulence in the TCV tokamak scrape-off layer, Plasma Phys. Control. Fusion, 47, L1 (2005).

[29] P. H. Diamond and T. S. Hahm, On the dynamics of turbulent transport near marginal stability Phys.Plasmas 23640.

[30] N. Yan, G. Xu, W. Zhang, J. Chang, L. Wang et al., Observation of Blobs and Holes in the Boundary Plasma of EAST Tokamak, Plasma Sci. Technol. 13410 (2011).

[31] P. Bak, C. Tang, and K. Wiesenfeld, Self-organized criticality, Phys. Rev. Lett. 59381 (1987).

[32] G. Boffetta, V. Carbone, P. Giuliani, P. Veltri,2 and A. Vulpiani, Phys. Rev. Lett. 83 4662 .

[33] Ph. Ghendrih et al., Transport barrier fluctuations governed by SOL turbulence spreading, J. Nucl. Mater. 363-365, 581 (2007).

[34] Ph. Ghendrih et al., Shearing effects on density burst propagation in SOL plasmas, J. Nucl. Mater. 390-391, 425 (2009).

[35] Y. Sarazin, Ph. Ghendrih, G. Attuel, C. Clément, X. Garbet et al., Theoretical understanding of turbulent transport in the SOL, J. Nucl. Mater. 313-316 796 (2003).

[36] W A Hornsby, M Siccinio, A G Peeters, E Poli, A P Snodin, F J Casson, Y Camenen and G Szepesi, Interaction of turbulence with magnetic islands: effect on bootstrap current, Plasma Phys. Control. Fusion 537, 054008 (2011).

[37] W. X. Wang, T. S. Hahm, W. W. Lee, G. Rewoldt, J. Manickam, and W. M. Tang, Nonlocal properties of gyrokinetic turbulence and the role of $E \times B$ flow shear, Phys. Plasmas 14072306 (2007).

[38] S.J. Allfrey, R. Hatzky, A.Bottino and L.Villard, Global Gyrokinetic Simulations of Nonlinear Interaction of Zonal Flows with ITG Modes, 29th EPS Conference ECA 26B, P-4.059 (2002).

[39] L. Villard et al., Full radius linear and nonlinear gyrokinetic simulations for tokamaks and stellarators: zonal flows, applied $E \times B$ flows, trapped electrons and finite beta, Nucl. Fusion 44, 172 (2004).

[40] G. Dif-Pradalier et al., On the validity of the local diffusive paradigm in turbulent plasma transport, Phys. Rev. E 82, 025401(R) (2010).

[41] Y. Sarazin, M. Bécoulet, P. Beyer, X. Garbet, Ph. Ghendrih et al., Edge issues in ITB plasmas in JET, Plasma Phys. Control. Fusion, 44, 2445 (2002).

[42] C. Figarella et al., Control of long range turbulent transport with biasing in the tokamak scrape-off-layer, J. Nucl. Mater. 337-339, 342, (2005).

[43] G. Ciraolo et al., Spreading of edge plasma turbulence in transport barriers, 33rd EPS Conference on Plasma Physics, ECA 30I, P-5.100 (2006).

[44] C. F. Figarella, S. Benkadda, P. Beyer, X. Garbet, and I. Voitsekhovitch, Transport Reduction by Rotation Shear in Tokamak-Edge Turbulence, Phys.Rev. Lett., 90015002 (2003).

[45] P. Beyer et al., Nonlinear Dynamics of Transport Barrier Relaxations in Tokamak Edge Plasmas, Phys. Rev. Lett. 94, 105001 (2005).

[46] G. Tresset, X. Litaudon, D. Moreau, X. Garbet and Contributors to the EFDAJETWork Programme $A$ dimensionless criterion for characterizing internal transport barriers in JET, Nucl. Fusion 42520 (2002).

[47] E. Floriani, R. Lima and D. Volchenkov, A toy model for a system at a threshold of stability, J. Phys. A : Math. Gen. 36, 4771 (2003). 
[48] Y. Pomeau and P. Manneville, Intermittent transition to turbulence in dissipative dynamical systems, Communications in Mathematical Physics 74, 189 (1980).

[49] G. Paladin and A. Vulpiani, Degrees of freedom of turbulence, Phys. Rev. A 35, R1971 (1987).

[50] B. Castaing, Y. Gagne, and E.J. Hopfinger, Velocity probability density-functions of high Reynolds-number turbulence, Physica D 46, 177 (1990).

[51] ICTR, A. Arneodo et al., Universal intermittent properties of particle trajectories in highly turbulent flows, Physical Review Letters Volume: 100, Issue: 25 (2008). 